\title{
Bryozoans from the lower Silurian (Wenlock) Steinsfjorden Formation of Ringerike, southern Norway
}

\author{
ANDREJ ERNST \& HANS ARNE NAKREM
}

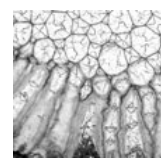

\begin{abstract}
A stenolaemate bryozoan fauna from the lower Silurian (Wenlock) Steinsfjorden Formation of the Ringerike district, southern Norway contains 10 species (2 cystoporates, 6 trepostomes, and 2 cryptostomes). Six species are new: Heterotrypa ringerikensis sp. nov., Trematopora maculata sp. nov., Amplexopora crassiparietum sp. nov., A. evae sp. nov., Orthopora worsleyi sp. nov., and Mediaporina kiaeri sp. nov. One species is described in open nomenclature: Eridotrypella sp. Trepostome bryozoans dominate this assemblage, both by diversity and abundance. The bryozoans occur in carbonates associated with evaporite minerals and sedimentary structures indicating very shallow depositional settings. $•$ Key words: lower Silurian, Bryozoa, Norway, taxonomy.
\end{abstract}

ERNST, A. \& NAKREM, H.A. 2015. Bryozoans from the lower Silurian (Wenlock) Steinsfjorden Formation of Ringerike, southern Norway. Bulletin of Geosciences 90(1), 65-87 (13 figures, 10 tables). Czech Geological Survey, Prague. ISSN 1214-1119. Manuscript received June 5, 2014; accepted in revised form September 11, 2014; published online December 3, 2014; issued January 26, 2015.

Andrej Ernst, Institut für Geologie, Universität Hamburg, Bundesstr. 55, D-20146 Hamburg, Germany; Andrej.Ernst@uni-hamburg.de•Hans Arne Nakrem, Natural History Museum (Geology), University of Oslo, P.O. Box 1172 Blindern,NO-0318Oslo, Norway; h.a.nakrem@nhm.uio.no

The purpose of this study is to describe the bryozoan fauna of the lower Wenlock Brattstad Member, Steinsfjorden Formation of the Ringerike district, Oslo Region, Norway (Fig. 1). The bryozoans present in these beds have been known since the work by Kiær (1908), but they have not until now been given a systematic treatment. The number of previously known species is low, and their distribution in Ringerike can add very little to the already established biostratigraphy of the area.

The Brattstad Member (upper "Etage" 9e and 9f of Kiær 1908) is $30 \mathrm{~m}$ thick in the studied areas of Nes bus stop, Ødegårdsviken and Åsa (Fig. 2). The lithology consists of medium to thickly bedded limestones with interbedded marls and dolomitic limestones. The limestones contain a relatively rich and varied fauna with thick massive favositid and oncolitic biostromes (the "Favosites Limestone" of Kiær 1908) near the top of the member from which the bryozoan material derives. Kiær (1908, p. 113) lists fossils from these beds ("Etage 9f") in various localities in the Ringerike district, but only one bryozoan taxon, "Monticuliporen [Amplexopora(?) sp.]", is mentioned. Whitaker (1977, pp. 24-26) also refers to the bryozoanrich beds as "polyzoan (Amplexopora)" beds. Spjeldnæs (1982) describes the bryozoan occurrences in these beds as "colonies ranging from only a few $\mathrm{cm}$ in diameter to small bioherms with three-dimensional frameworks". Bryozoans are even found in the lowermost levels of the overlying red beds of the Ringerike Group (late Wenlock to Ludlow age). The age of the bryozoan beds are Sheinwoodian to Homerian as based on occurrences of ostracodes and brachiopods as well as agnathans and thelodonts (on directly overlying beds) (Worsley et al. 1983, 2011). Geochemical analyses of interbedded bentonites (Hetherington et al. 2011) support these datings.

\section{Depositional environment}

Alternations of marls, red argillaceous dolomites, dolomitic limestones, and limestones, with occurrence of oosparite, oncolites, algal laminated dolomitic limestones with mud cracks, stromatolites as well as evaporitic minerals (Fig. 3B) indicate developments of shallow subtidal to supratidal environments (Olaussen 1981). The fossil components are characterized by many individuals belonging to few taxa, notably gastropods, ostracods, brachiopods and rare trilobites (Kiær 1908, Olaussen 1981). Tabulate corals (Fig. 3C) and echinoderms are locally present.

The common microfacies are represented by floatstones with abundant bryozoans (mainly trepostomes (Fig. 3A, F), locally poorly washed rudstones (Fig. 3E), and bryozoan and algal bindstones (Fig. 3B-D). The matrix is usually micrite-rich, containing various skeletal grains (Fig. 3G). Calcimicrobes are common on some localities, 

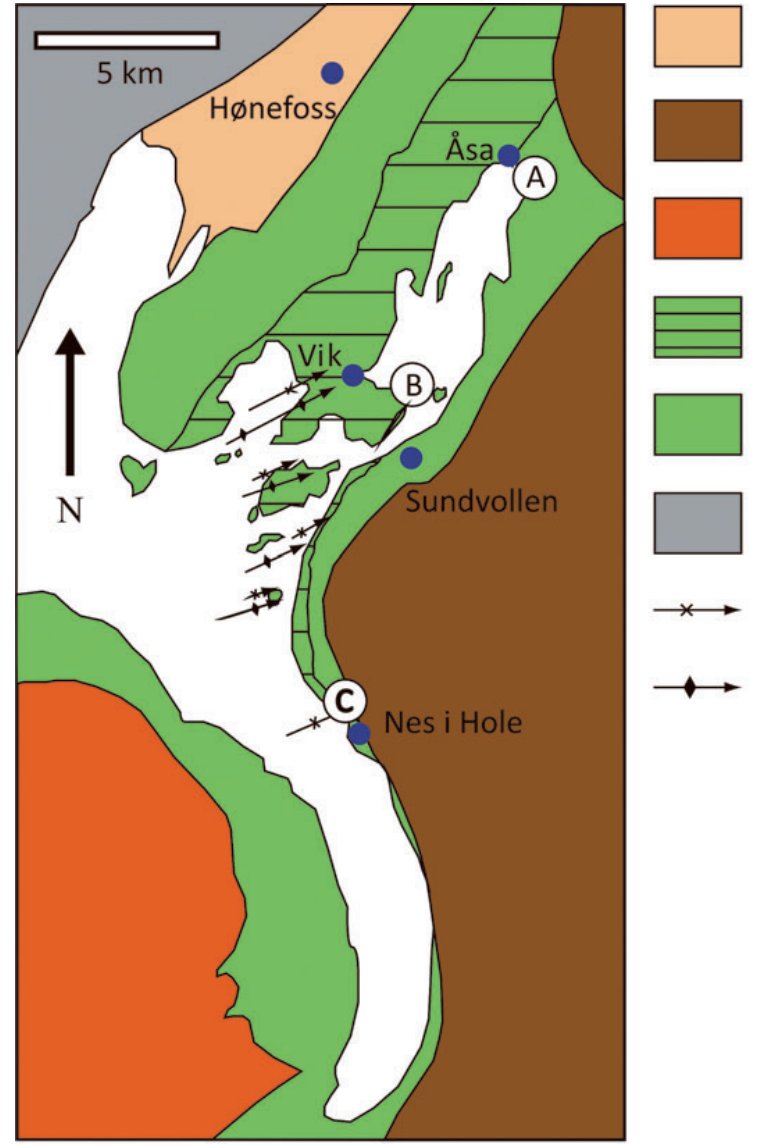

Quaternary cover

Upper Carboniferous to Lower Permian volcano-sedimentary rocks

Upper Carboniferous to Lower Permian intrusive rocks

Marine Silurian rocks

Lower Palaeozoic sedimentary rocks

Proterozoic crystalline basement

Synclinal fold axis with plunge

Anticlinal fold axis with plunge
Figure 1. Map of the Ringerike District showing sample localities and major structural features. (A) - Åsa locality, (B)- Ødegårdsviken locality, (C) - Nes bus stop locality. represented by various Girvanella and Rothpletzella species. Microbial crusts are common, often forming oncoids (Fig. 3C).

The faunal composition and microfacies characteristics are reduced due to stressed conditions in an environment with fluctuating salinities.

\section{Material and methods}

Bryozoan samples were collected from the localities Nes bus stop, Ødegårsviken and Åsa (see Fig. 1). The investigated bryozoans were studied from thin sections using a transmitted light binocular microscope. 107 oriented and non-oriented thin sections were used. The material is housed at the Natural History Museum (Geology), Oslo, under numbers PMO 221.750-221.841.

Morphological character terminology is adopted from

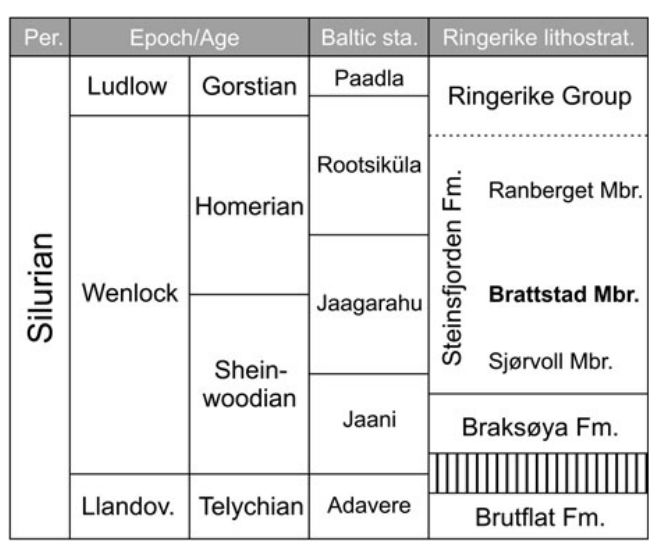

Figure 2. Wenlock stratigraphy of the Ringerike District (based on Melchin et al. 2012; Worsley et al. 1983, 2011).

Anstey \& Perry (1970) for trepostomes, and Hageman (1993) for cryptostomes. The following morphologic characters were measured for statistical use (Fig. 4): Branch Width, Branch Thickness, Exo- (Endo-) zone Width, Autozooecial Aperture Width, Autozooecial Aperture Spacing (Along Branch/diagonally for cryptostomes), Acanthostyle Diameter, Lunarium Width/Length/Thickness, Vesicle Diameter/Spacing/Number per Aperture, Wall Thickness in Exozone, and Macular Diameter (Spacing), Autozooecial Diaphragm Spacing, Meso- (Exila-, Hetero-) zooecia Width, Mesozooecial Diaphragm Spacing.

The spacing of structures was measured as the distance between centres. Additional quantitative characters include the Number of Mesozooecia, Exilazooecia and Acanthostyles surrounding each autozooecial aperture. Statistics were summarized using arithmetic mean, sample standard deviation, coefficient of variation, and minimum and maximum values.

Figure 3. Carbonate microfacies of the Steinsfjorden Formation of Ringerike, southern Norway. • A - bryozoan floatstone, Nes bus stop, PMO 221.766. - B - bryozoan bindstone (Trematopora maculata, Fistulipora nummulina). Arrow - relicts of gypsum crystals, Ødegårdsviken, PMO 221.820. - C - coral-oncolitic floatstone, Åsa, PMO 221.813. • D - bryozoan bindstone (Trematopora maculata, Fistulipora nummulina), Ødegårdsviken, PMO 221.824. $\bullet$ E - poorly washed rudstone, Ødegårdsviken, PMO 221.779. • F - bryozoan floatstone, Ødegårdsviken, PMO 221.792. • G - matrix, Nes bus stop, PMO 221.766. 
Andrej Ernst \& Hans Arne Nakrem • Bryozoans from the lower Silurian (Wenlock) Steinsfjorden Formation of Ringerike
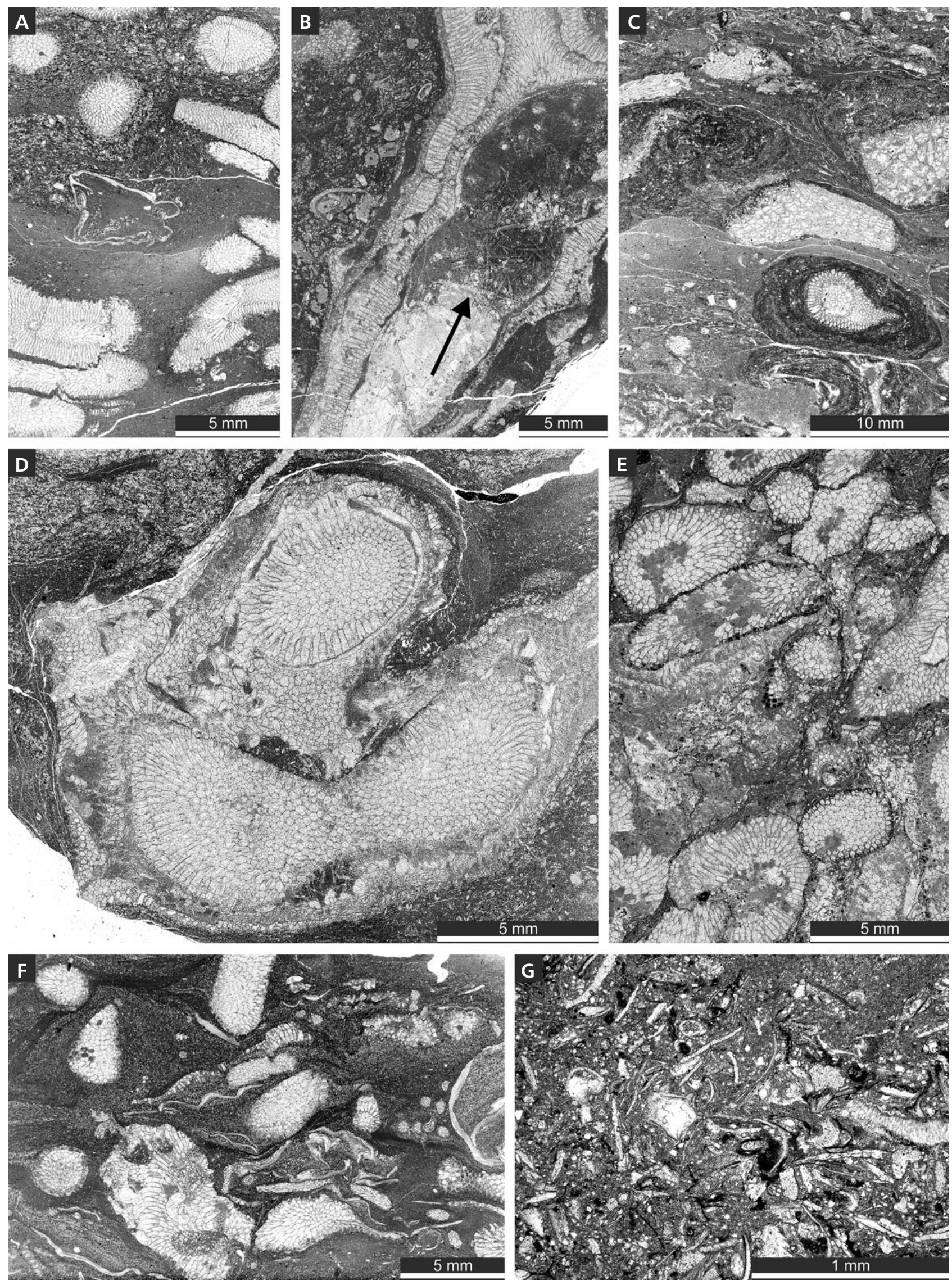


\section{Results and discussion}

The bryozoan colonies are generally well preserved and show little signs of abrasion. Large, thin branches up to $5 \mathrm{~mm}$ in diameter are often not broken, the whole fauna has only been transported a short distance, and they may represent time intervals of more normal marine conditions. However, they are not considered preserved in situ. The bryozoans are therefore interpreted as typical for the depositional environment in which the sediments were formed. The three bryozoans species previously known have a Wenlock or Ludlow stratigraphic distribution elsewhere. The new species described herein have many similarities with species known from time equivalent strata in Europe and the USA, but only further research may reveal their biostratigraphic as well as biogeographic value.

\section{Systematic palaeontology}

Phylum Bryozoa Ehrenberg, 1831

Class Stenolaemata Borg, 1926

Order Cystoporata Astrova, 1964

Family Fistuliporidae Ulrich, 1882

\section{Genus Fistulipora M'Coy, 1849}

Type species. - Fistulipora minor M'Coy, 1849. Carboniferous; England.

Diagnosis. - Massive, encrusting or ramose colonies. Cylindrical autozooecia with thin walls and complete diaphragms. Apertures rounded, possessing horse-shoe shaped lunaria. Autozooecia separated by the extrazooecial vesicular skeleton.

Comparison. - Fistulipora M'Coy, 1849 differs from Eridopora Ulrich, 1882 in having rounded, horseshoe-shaped lunaria instead of triangular ones. Furthermore, Eridopora develops persistently encrusting colonies, whereas Fistulipora may also develop massive and branched colonies.

Occurrence. - Ordovician to Permian; worldwide.

\section{Fistulipora nummulina Nicholson \& Foord, 1885}

Figure 5A-E, Table 1

1885 Fistulipora nummulina Nicholson \& Foord, pp. 506-507, text-fig. 4, pl. 15, figs 2-2b.

1885 Fistulipora dobunica Nicholson \& Foord, p. 511, pl. 17, figs 3-3b.

1969 Fistulipora nummulina Nicholson \& Foord, 1885. Owen, pp. 627-628, pl. 114, figs 1, 2.
Table 1. Measurements of Fistulipora nummulina Nicholson \& Foord, 1885. Abbreviations: $\mathrm{N}$ - number of measurements; X - mean; SD - standard deviation; CV - coefficient of variation; MIN - minimal value; $\mathrm{MAX}$ - maximal value.

\begin{tabular}{lcccccc}
\hline & $\mathrm{N}$ & $\mathrm{X}$ & $\mathrm{SD}$ & $\mathrm{CV}$ & $\mathrm{MIN}$ & $\mathrm{MAX}$ \\
\hline Aperture width, mm & 33 & 0.16 & 0.027 & 16.46 & 0.12 & 0.22 \\
Aperture spacing, mm & 31 & 0.24 & 0.035 & 14.41 & 0.17 & 0.32 \\
Vesicle width, mm & 23 & 0.10 & 0.031 & 32.55 & 0.03 & 0.15 \\
Vesicle spacing, mm & 25 & 0.10 & 0.028 & 28.98 & 0.06 & 0.15 \\
Vesicles per aperture & 8 & 6.0 & 1.414 & 23.57 & 4.0 & 8.0 \\
\hline
\end{tabular}

Material. - PMO 221.756, PMO 221.758, PMO 221.790, PMO 221.764, PMO 221.817, PMO 221.820, PMO 221.822, PMO 221.824, PMO 221.827, PMO 221.829.

Description. - Encrusting colonies, $0.25-0.85 \mathrm{~mm}$ in thickness. Autozooecia growing from 0.002-0.008 m thick epitheca, bending sharply at their bases towards colony surface. Autozooecial apertures circular to oval. Basal diaphragms rare to absent. Lunaria well developed, horseshoe shaped to slightly triangular, $0.06-0.08 \mathrm{~mm}$ wide, 0.03-0.04 mm long and 0.03-0.04 mm thick. Vesicles abundant, 4-8 surrounding each autozooecial aperture, irregularly shaped, medium in size, often large at the base, polygonal in tangential section, box-like to hemispheric, with plane or concave roofs in exozone. Autozooecial walls granular, 0.003-0.005 mm thick. Extensive extrazooecial material in exozone developed. Maculae not observed.

Remarks. - Fistulipora nummulina Nicholson \& Foord, 1885 is similar to $F$. parva Hennig, 1908 from the Wenlock of Gotland, but differs by smaller autozooecial apertures (aperture width $0.12-0.22 \mathrm{~mm}$ vs $0.22-0.25 \mathrm{~mm}$ in $F$. par$v a)$. Fistulipora nummulina differs from $F$. ternavensis Astrova, 1965 from the lower Silurian (Wenlock) of Moldova in smaller and closer spaced autozooecial apertures (aperture width $0.12-0.22 \mathrm{~mm}$ vs $0.18-0.23 \mathrm{~mm}$ in F. ternavensis).

Occurrence. - Much Wenlock Limestone Formation, Silurian, Wenlock, Homerian; Dudley, England. Steinsfjorden Formation, Brattstad Member, Silurian, Wenlock, Sheinwoodian-Homerian; Nes bus stop, Ringerike, Norway.

Fistulipora crustula Bassler, 1906

Figures 5F-J, 6A, B, Table 2

1906 Fistulipora crustula Bassler, p. 24, pl. 7, figs 7-10, pl. 8 , figs 16,17 , pl. 23, fig. 15 .

Material. - PMO 221.775-221.776. 


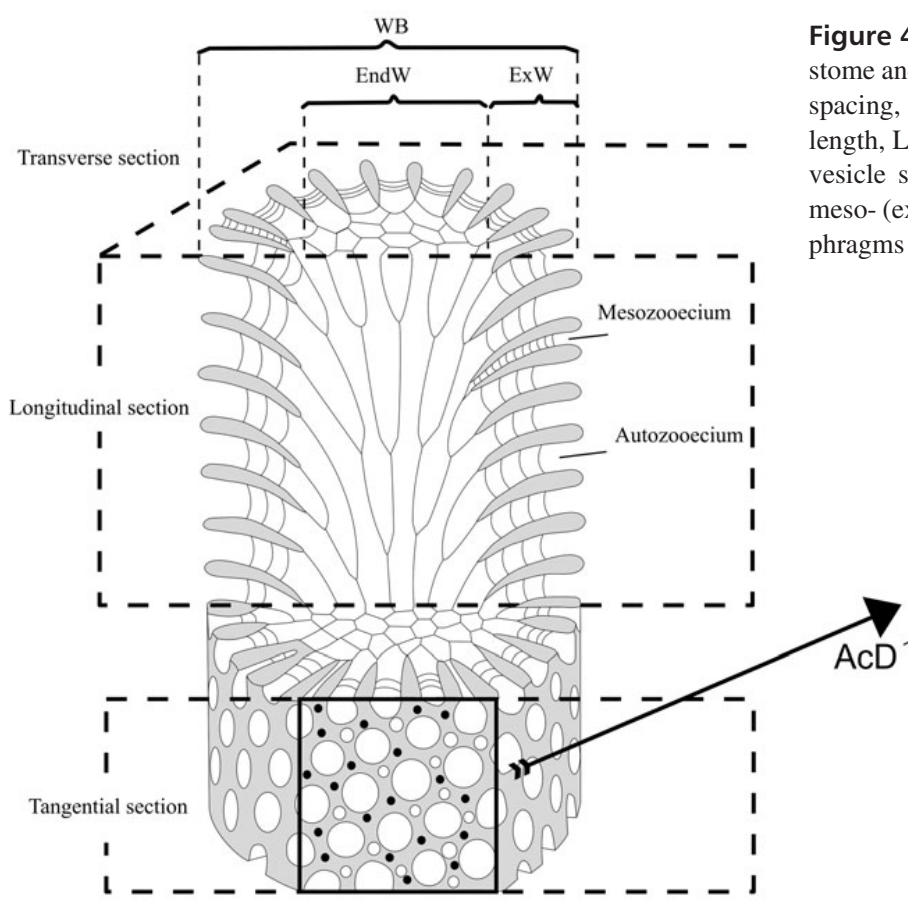

Figure 4. Some important measurements made on the trepostome and cystoporate bryozoans. Abbreviations: ADB - aperture pacing, AW - autozooecial aperture width, LuL - lunarium ength, LuW - lunarium width, LuT - lunarium thickness, VSp vesicle spacing, DSp -autozooecial diaphragm spacing, MD meso- (exila-, hetero-) zooecial width, MDSp - mesozooecial diahragms spacing, AcD - acanthostyles diameter.

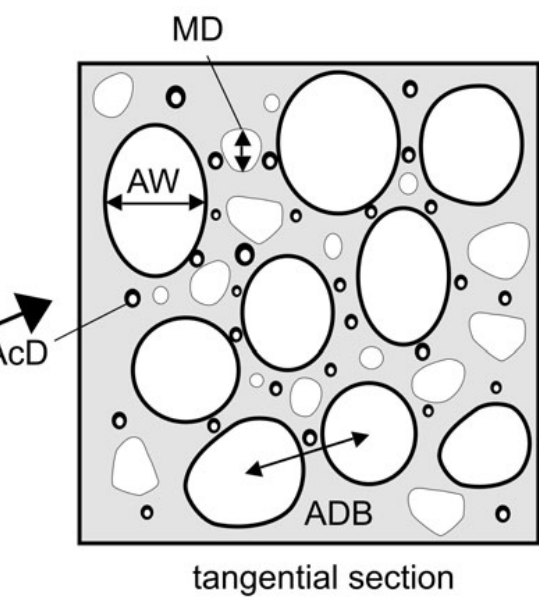

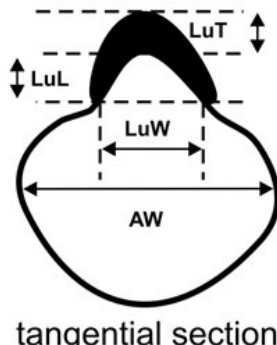

tangential section

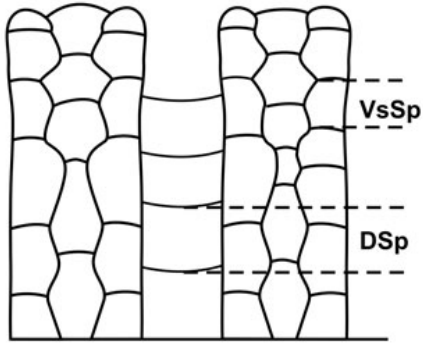

longitudinal section

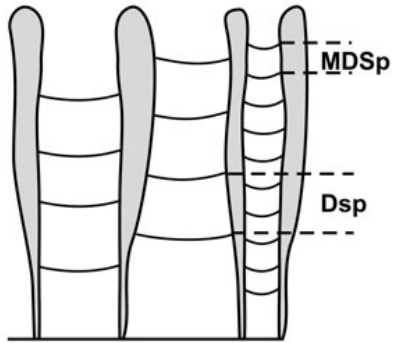

Description. - Encrusting colonies, $0.65-1.38 \mathrm{~mm}$ in thickness. Autozooecia growing from $0.015-0.030 \mathrm{~m}$ thick epitheca, bending sharply at their bases towards colony surface. Autozooecial apertures circular to oval. Basal diaphragms rare to common, thin, deflected proximally. Lunaria well developed, horseshoe shaped. Vesicles irregularly shaped, medium in size, polygonal in tangential section, box-like to hemispheric, with concave roofs in exozone, 5-11 surrounding each autozooecial aperture. Autozooecial walls granular, 0.003-0.005 mm thick. Maculae consisting of vesicular skeleton common, $0.30-0.40 \mathrm{~mm}$ in diameter. Macrozooecia locally occurring.

Remarks. - Fistulipora crustula Bassler, 1906 is similar to F. crustuliformis Astrova, 1959 from the upper Silurian (Ludlow) of Tuva (Russia), but differs from the latter in smaller autozooecia (aperture width $0.18-0.25 \mathrm{~mm}$ vs $0.22-0.26 \mathrm{~mm}$ in $F$. crustuliformis).

Occurrence. - Rochester Shale, Wenlock, lower Silurian; USA. Steinsfjorden Formation, Brattstad Member, Silur- ian, Wenlock, Sheinwoodian-Homerian; Ødegårdsviken, Ringerike, Norway.

Order Trepostomata Ulrich, 1882

Suborder Halloporina Astrova, 1965

Family Heterotrypidae Ulrich, 1890

\section{Genus Heterotrypa Nicholson, 1879}

Type species. - Monticulipora frondosa d'Orbigny, 1850. Upper Ordovician (Cincinnatian); North America.

Diagnosis. - Frondose, ramose or less commonly encrusting colonies. Autozooecial walls variably thick. Zooecial boundaries form a conspicuous dark line in inner exozones and in a broad zone of abutting laminae or are completely obscured in outer exozones. Walls generally amalgamated. Diaphragms are generally few in endozones, but are moderately abundant in some species. In exozones, autozooecial diaphragms are closely and regularly spaced, thin, planar and perpendicular to 
Table 2. Measurements of Fistulipora crustula Bassler, 1906. Abbreviations as in Table 1.

\begin{tabular}{lcccccc}
\hline & $\mathrm{N}$ & $\mathrm{X}$ & $\mathrm{SD}$ & $\mathrm{CV}$ & $\mathrm{MIN}$ & $\mathrm{MAX}$ \\
\hline Aperture width, mm & 25 & 0.21 & 0.019 & 8.95 & 0.18 & 0.25 \\
Aperture spacing, mm & 25 & 0.25 & 0.043 & 17.02 & 0.18 & 0.35 \\
Vesicle width, mm & 25 & 0.07 & 0.023 & 31.10 & 0.03 & 0.13 \\
Vesicle spacing, mm & 25 & 0.08 & 0.020 & 24.97 & 0.04 & 0.11 \\
Vesicles per aperture & 6 & 8.2 & 2.041 & 24.99 & 5.0 & 11.0 \\
Lunarium width, mm & 7 & 0.15 & 0.035 & 23.41 & 0.11 & 0.20 \\
Lunarium length, mm & 7 & 0.035 & 0.012 & 33.35 & 0.025 & 0.050 \\
Lunarium thickness, mm & 7 & 0.033 & 0.014 & 43.25 & 0.015 & 0.050 \\
Macrozooecia aperture width, mm & 10 & 0.30 & 0.019 & 6.41 & 0.28 & 0.33
\end{tabular}

the zooecial walls. Intermonticular mesozooecia range from abundant and regularly arranged to scattered or absent. Mesozooecia commonly develop moniliform chambers at proximal ends and tend to become smaller or are terminated distally within exozones. Diaphragms in mesozooecia noticeably thicker and more closely spaced than autozooecial diaphragms. Acanthostyles common, small to moderately large, occurring both in endozone and exozone. Monticules generally have a central cluster of a few mesozooecia.

Remarks. - Heterotrypa differs from the most similar genus Dekayia Milne-Edwards \& Haime, 1851 by having fewer and smaller acanthostyles as well as more abundant mesozooecia.

Occurrence. - Ordovician to Devonian; worldwide.

\section{Heterotrypa ringerikense sp. nov.} Figures 6C-F, 7A-E, Table 3

Etymology. - The species has its name after Ringerike, an area SW of the Oslo Region, which is famous for its rich $\mathrm{Si}$ lurian bryozoan-bearing rocks.

Holotype. - PMO 202.721B.

Paratypes. - PMO 221.763, PMO 221.773-221.777, PMO 221.785-221.786, PMO 221.799, PMO 202.721(A, C-E).

Type locality. - Ødegårdsviken, Ringerike, Norway.

Type stratum. - Steinsfjorden Formation, Brattstad Member, Silurian, Wenlock, Sheinwoodian-Homerian.
Diagnosis. - Ramose branched colonies; secondary overgrowth common; autozooecial diaphragms rare to common, thin; mesozooecia rare to common; acanthostyles moderately large, 2-7 surrounding each autozooecial aperture; macroacanthostyles present; maculae consisting of macrozooecia.

Description. - Ramose branched colonies, branch diameter 2.4-5.0 mm. Exozone distinct, 0.45-1.25 mm wide, endozone $1.30-2.88 \mathrm{~mm}$ wide. Secondary overgrowth common, $0.65-1.00 \mathrm{~mm}$ in thickness. Autozooecia long, polygonal in cross section in endozone, bending sharply in exozone. Autozooecial apertures polygonal. Autozooecial diaphragms rare to common, thin, concentrated mainly in the transition between exoand endozones. Mesozooecia rare to common, originating at base of exozone, beaded in places of development of diaphragms. Diaphragms in mesozooecia straight. Acanthostyles moderately large, prominent, having distinct hyaline cores, 2-7 surrounding each autozooecial aperture. Macroacanthostyles locally present. Autozooecial walls $0.005-0.010 \mathrm{~mm}$ thick, granular-prismatic in endozone; showing reversal $\mathrm{V}$-shaped lamination, integrated with locally visible serrated dark border between zooecia, locally weakly monilae-shaped thickened, 0.013-0.050 mm thick in exozone. Maculae consisting of macrozooecia.

Remarks. - Heterotrypa ringerikense sp. nov. differs from H. enormis Astrova, 1959 from the upper Silurian (Ludlow) of western Tuva (Russia) by larger and more abundant acanthostyles (acanthostyle diameter 0.025-0.065 $\mathrm{mm} v \mathrm{~s}$ $0.03 \mathrm{~mm}$ in $H$. enormis; 2-7 acanthostyles around each aperture vs 3-4 in $H$. enormis). Heterotrypa ringerikense sp. nov. differs from H. ovata Astrova, 1957 from the upper Silurian (Ludlow) of western Tuva (Russia) by less abundant mesozooecia and by larger and more abundant acanthostyles (2-7 acanthostyles around each aperture $v s$ 1-2 in H. ovata).

Occurrence. - Steinsfjorden Formation, Brattstad Member, Silurian, Wenlock, Sheinwoodian-Homerian; Ødegårdsviken, Ringerike, Norway.

\section{Genus Asperopora Owen, 1969}

Type species. - Callopora aspera Hall, 1852. Silurian (Wenlock); USA (New York), England.

Figure 5. A-E - Fistulipora nummulina Nicholson \& Foord, 1885. • A, B - encrusting colony, PMO 221.764. • C - longitudinal section, PMO 221.820. - D - tangential section, PMO 221.817. • E - tangential section, PMO 221.764. • F-J - Fistulipora crustula Bassler, 1906. $\bullet$ F, G - longitudinal section, PMO 221.775. • H - longitudinal section, PMO 221.776. • I, J - tangential section, PMO 221.775. 

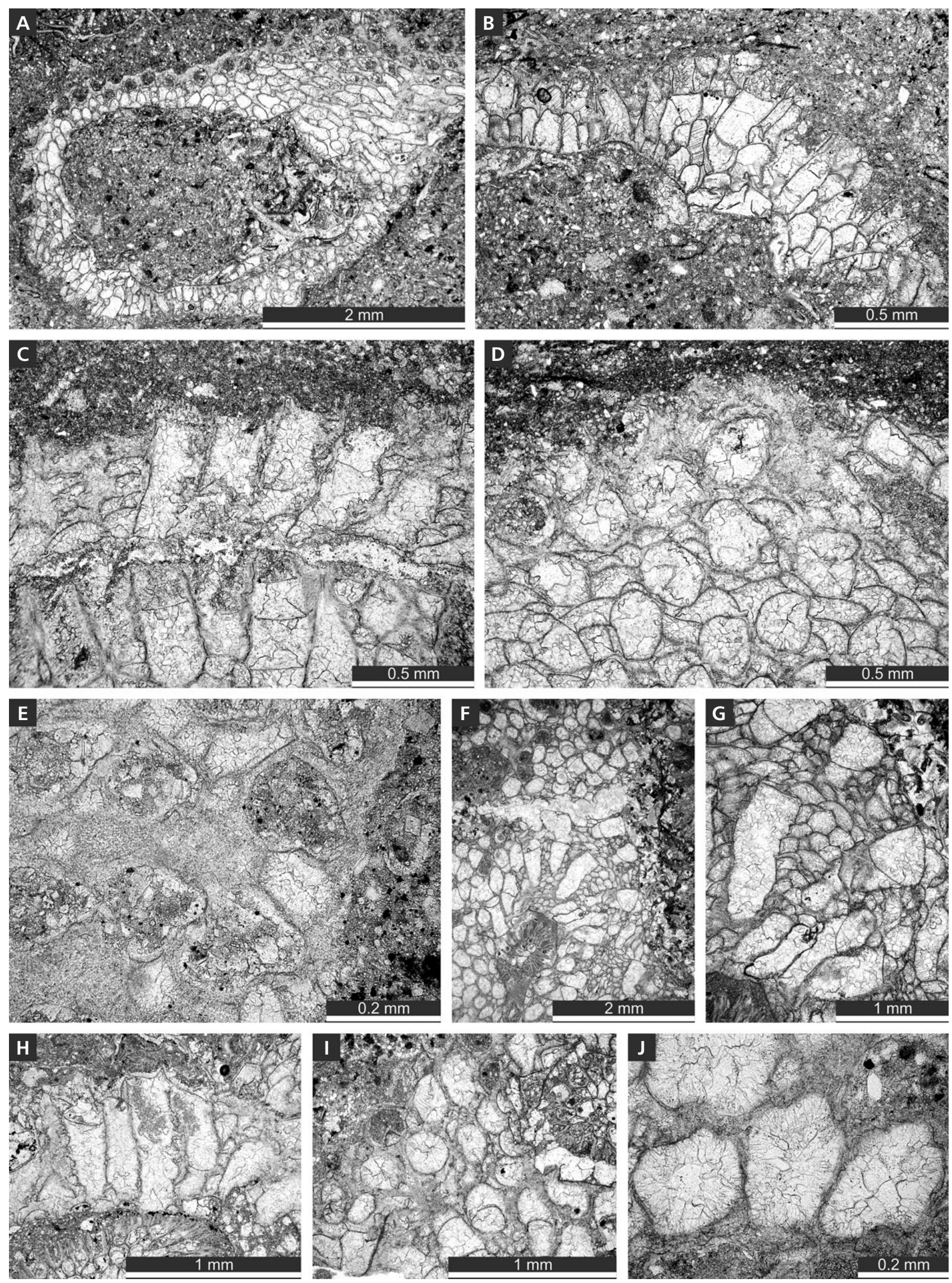
Table 3. Measurements of Heterotrypa ringerikense sp. nov. Abbreviations as in Table 1.

\begin{tabular}{lcccccc}
\hline & $\mathrm{N}$ & $\mathrm{X}$ & $\mathrm{SD}$ & $\mathrm{CV}$ & MIN & MAX \\
\hline Branch width, mm & 6 & 3.50 & 1.172 & 33.51 & 2.40 & 5.00 \\
Exozone width, mm & 6 & 0.74 & 0.312 & 42.08 & 0.45 & 1.25 \\
Endozone width, mm & 6 & 2.01 & 0.612 & 30.40 & 1.30 & 2.88 \\
Aperture width, mm & 55 & 0.17 & 0.025 & 15.13 & 0.11 & 0.22 \\
Aperture spacing, mm & 55 & 0.20 & 0.031 & 14.94 & 0.15 & 0.29 \\
Aperture width, mm (macular) & 20 & 0.24 & 0.025 & 10.47 & 0.2 & 0.28 \\
Aperture spacing, mm (macular) & 20 & 0.30 & 0.044 & 14.63 & 0.24 & 0.42 \\
Mesozooecia width, mm & 50 & 0.06 & 0.021 & 37.98 & 0.03 & 0.10 \\
Acanthostyle diameter, mm & 45 & 0.044 & 0.009 & 20.22 & 0.025 & 0.065 \\
Macroacanthostyle diameter, mm & 20 & 0.067 & 0.012 & 17.22 & 0.043 & 0.088 \\
Exozonal wall thickness, mm & 45 & 0.030 & 0.010 & 31.96 & 0.013 & 0.050 \\
Acanthostyles per aperture & 45 & 3.7 & 1.424 & 38.38 & 2.0 & 7.0 \\
$\begin{array}{l}\text { Mesozooecial diaphragms spacing, } \\
\text { mm }\end{array}$ & 10 & 0.10 & 0.025 & 25.40 & 0.05 & 0.14 \\
\hline
\end{tabular}

Diagnosis. - Colonies incrusting, massive or branched ramose; autozooecia tubular; autozooecial apertures rounded to petaloid due to inflecting acanthostyles; autozooecial diaphragms few to many; mesozooecia abundant, originating in exozones, sometimes cystose, containing thin diaphragms; acanthostyles commonly large and abundant, with clear cores, inflecting autozooecial apertures; autozooecial walls moderate in thickness, finely laminated, merged, irregularly thickened in exozone (modified after Owen 1969).

Remarks. - Asperopora Owen, 1969 is similar to Leioclema Ulrich, 1882 but differs by irregularly thickened walls in exozone. In general, Asperopora has thinner walls and smaller acanthostyles. Asperopora differs from Trematopora Hall, 1852 by having mesozooecia not sealed by calcitic material. Moreover, Trematopora develops exceptionally ramose-branched colonies, whereas Asperopora forms also encrusting and massive ones.

Occurrence. - Upper Ordovician to upper Silurian; Europe, USA.

\section{Asperopora bellum (Pushkin, 1976)}

Figure 7F-I, Table 4

1976 Lioclema bellum Pushkin, pp. 30-31, text-fig. 13, pl. 11 , fig. 3 .
Material. - PMO 221786-221.787, PMO 221.793, PMO 221.798, PMO 221.800.

Description. - Encrusting colonies, 0.7-1.0 mm thick. Autozooecia budding from a thin epitheca, growing a short distance parallel to the substrate, then bending sharply to the colony surface. Autozooecial apertures roundedpolygonal. Autozooecial diaphragms common, straight, thin. Acanthostyles abundant, 1-3 surrounding each aperture, originating at the base of exozone, having distinct calcite cores and dark, laminated sheaths, deeply indenting into autozooecial space. Mesozooecia abundant, 6-11 surrounding each autozooecial aperture, rounded-polygonal in transverse section, originating at the base of exozone. Autozooecial walls laminated, merged, 0.020-0.050 mm thick. Maculae not observed.

Remarks. - Asperopora bellum (Pushkin, 1976) differs from A. asperum (Hall, 1852) in larger autozooecial apertures (average aperture width $0.14 \mathrm{~mm} v s 0.12 \mathrm{~mm}$ in A. asperum), and in less abundant mesozooecia (6-11 mesozooecia per aperture vs 9-14 in A. asperum). Asperopora bellum differs from $A$. multipora (Bassler, 1906) from the Rochester Shale of USA in smaller autozooecial apertures (aperture width $0.10-0.18 \mathrm{~mm}$ vs $0.15-0.20 \mathrm{~mm}$ in $A$. multipora), and fewer acanthostyles (1-3 vs 4-7 per aperture in A. multipora).

Occurrence. - Ludlow, Silurian; Brest Depression, Belarus. Steinsfjorden Formation, Brattstad Member, Silurian, Wenlock, Sheinwoodian-Homerian; Ødegårdsviken, Ringerike, Norway.

Family Trematoporidae Miller, 1889

\section{Genus Trematopora Hall, 1852}

Type species. - Tretmatopora tuberculosa Hall, 1852; lower Silurian (Niagaran); North America.

Diagnosis. - Ramose colonies, often originating from encrusting base. Autozooecial apertures oval to rounded with peristomes. Diaphragms usually rare, often absent in endozone. Abundant mesozooecia with abundant diaphragms, thin-walled and beaded in initial parts of exozone, near colony surface becoming thick-walled. Mesozooecial apertures often completely covered by laminated skeleton. Acanthostyles abundant. Walls thin in endozone, thickened in

Figure 6. A, B - Fistulipora crustula Bassler, 1906, tangential section, PMO 221.775. • C-F - Heterotrypa ringerikensis sp. nov.; C, D - longitudinal section, holotype PMO 202.721B; E - transverse section, holotype PMO 202.721B; F, G - longitudinal section of encrusting colony, paratype PMO 202.721A. 

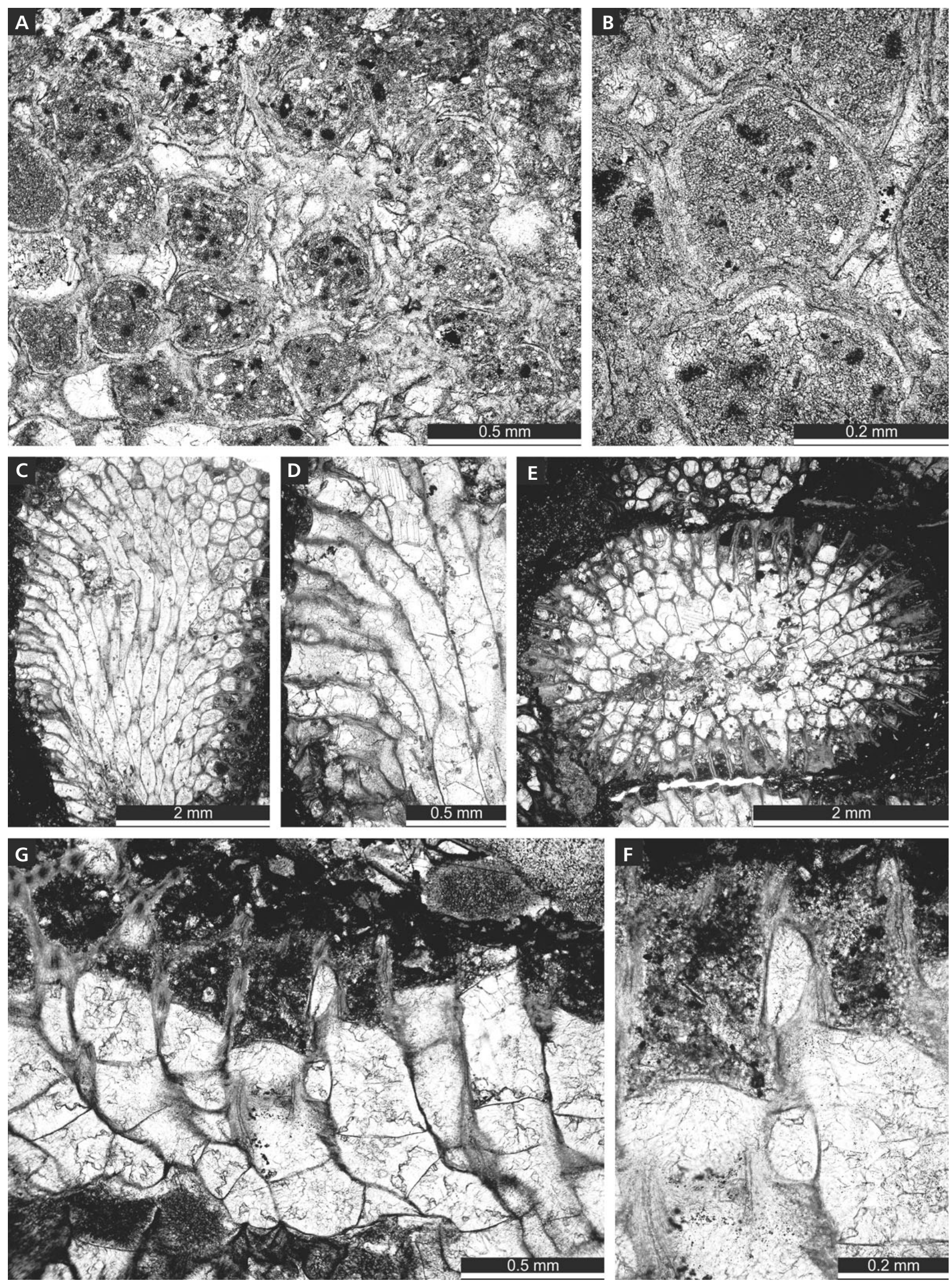
Table 4. Measurements of Asperopora bellum (Pushkin, 1976). Abbreviations as in Table 1 .

\begin{tabular}{lcccccc}
\hline & $\mathrm{N}$ & $\mathrm{X}$ & $\mathrm{SD}$ & $\mathrm{CV}$ & $\mathrm{MIN}$ & $\mathrm{MAX}$ \\
\hline Aperture width, mm & 36 & 0.14 & 0.023 & 16.74 & 0.10 & 0.18 \\
Aperture spacing, mm & 36 & 0.20 & 0.031 & 15.50 & 0.14 & 0.26 \\
Mesozooecia width, mm & 36 & 0.05 & 0.015 & 32.18 & 0.02 & 0.07 \\
Mesozooecia per aperture & 30 & 7.4 & 1.329 & 17.96 & 6.0 & 11.0 \\
Acanthostyle diameter, mm & 36 & 0.037 & 0.007 & 17.90 & 0.025 & 0.060 \\
$\begin{array}{l}\text { Acanthostyles per aperture } \\
\text { Mesozooecial diaphragms }\end{array}$ & 30 & 2.3 & 0.596 & 25.91 & 1.0 & 3.0 \\
spacing, mm & 21 & 0.09 & 0.029 & 30.43 & 0.06 & 0.17 \\
\hline
\end{tabular}

Table 5. Measurements of Trematopora maculata sp. nov. Abbreviations as in Table 1.

\begin{tabular}{|c|c|c|c|c|c|c|}
\hline & $\mathrm{N}$ & $\mathrm{X}$ & SD & $\mathrm{CV}$ & MIN I & MAX \\
\hline Branch width, mm & 10 & 3.7 & 0.685 & 18.61 & 2.7 & 4.6 \\
\hline Exozone width, mm & 10 & 0.8 & 0.198 & 25.28 & 0.4 & 1.1 \\
\hline Endozone width, mm & 10 & 2.1 & 0.364 & 17.21 & 1.4 & 2.6 \\
\hline Aperture width, mm & 79 & 0.16 & 0.025 & 15.43 & 0.11 & 0.22 \\
\hline Aperture spacing, mm & 79 & 0.22 & 0.036 & 16.73 & 0.15 & 0.36 \\
\hline $\begin{array}{l}\text { Aperture width, } \mathrm{mm} \\
\text { (macular) }\end{array}$ & 15 & 0.25 & 0.025 & 9.74 & 0.23 & 0.33 \\
\hline $\begin{array}{l}\text { Aperture spacing, } \mathrm{mm} \\
\text { (macular) }\end{array}$ & 15 & 0.33 & 0.058 & 17.69 & 0.27 & 0.52 \\
\hline Mesozooecia width, mm & 70 & 0.065 & 0.019 & 28.76 & \multicolumn{2}{|c|}{0.0330 .120} \\
\hline Mesozooecia per aperture & 30 & 2.9 & 0.960 & 33.09 & 1.0 & 4.0 \\
\hline Acanthostyle diameter, mm & 70 & 0.052 & 0.010 & 19.77 & 0.030 & 0.088 \\
\hline Acanthostyles per aperture & 70 & 2.7 & 0.823 & 30.47 & 1.0 & 4.0 \\
\hline $\begin{array}{l}\text { Mesozooecial diaphragms } \\
\text { spacing, mm }\end{array}$ & 70 & 0.10 & 0.024 & 23.39 & 0.06 & 0.19 \\
\hline $\begin{array}{l}\text { Autozooecial diaphragms } \\
\text { spacing, } \mathrm{mm}\end{array}$ & 30 & 0.16 & 0.063 & 38.50 & 0.10 & 0.30 \\
\hline $\begin{array}{l}\text { Exozonal wall thickness, } \\
\mathrm{mm}\end{array}$ & 50 & 0.037 & 0.010 & 27.07 & 0.018 & 0.058 \\
\hline
\end{tabular}

peripheral parts of exozone displaying obliquely laminated microstructure.

Remarks. - Trematopora Hall, 1852 differs from Batostoma Ulrich, 1882 by having oval to rounded autozooecial apertures and abundant mesozooecia covered with skeletal material, from Eridotrypa Ulrich, 1893 by having autozooecia that bend sharply in exozone, possess rounded apertures and are arranged irregularly on the colony surface, as well as by abundant acanthostyles.
Occurrence. - Ordovician to Silurian, worldwide.

\section{Trematopora maculata sp. nov.}

Figure $8 \mathrm{~A}-\mathrm{H}$, Table 5

Etymology. - The species is named after presence of maculae of macrozooecia.

Holotype. - PMO 221.816.

Paratypes. - PMO 221.783-PMO 221.784, PMO 221.786-PMO 221.787, PMO 221.791, PMO 221.799, PMO 221.815, PMO 221.817-221.841.

Type locality. - Ødegårdsviken, Ringerike, Norway.

Type stratum. - Steinsfjorden Formation, Brattstad Member, Silurian, Wenlock, Sheinwoodian-Homerian.

Diagnosis. - Branched colonies with distinct exozones; secondary overgrowth common; autozooecial diaphragms rare to common; mesozooecia common to abundant in exozone, beaded, 2-4 surrounding each autozooecial aperture; acanthostyles moderately large, 1-4 surrounding each autozooecial aperture; autozooecial walls laminated with locally visible zooecial border, locally monilae-shaped thickened, maculae of few macrozooecia.

Description. - Ramose branched colonies, branch diameter 2.7-4.6 mm. Exozone distinct, 0.4-1.1 mm wide, endozone 1.4-2.6 mm wide. Secondary overgrowth common. Autozooecia long, polygonal in cross section in endozone, bending sharply in exozone. Autozooecial apertures rounded to slightly angular. Autozooecial diaphragms rare to common, thin, concentrated mainly in the transition between exo- and endozones. Mesozooecia common to abundant, originating at base of exozone, beaded in places of development of diaphragms, 2-4 surrounding each autozooecial aperture. Diaphragms in mesozooecia straight, abundant. Acanthostyles moderately large, prominent, having distinct hyaline cores, 1-4 surrounding each autozooecial aperture. Autozooecial walls 0.003-0.005 mm thick, granular-prismatic in endozone; showing reversal V-shaped lamination, integrated with locally visible dark border between zooecia, locally monilae-shaped thickened, 0.02-0.06 mm thick in exozone. Maculae formed by few macrozooecia.

Figure 7. A-E-Heterotrypa ringerikensis sp. nov.; A, B - tangential section, paratype PMO 202.721A.; C, D - branch transverse section, paratype PMO 221.763.; E - tangential section showing macroacanthostyles, paratype PMO 202.721E. • F-I - Asperopora bellum (Pushkin, 1976), PMO 221.793; F - tangential section; G, H - longitudinal section; I - tangential section. $\bullet \mathrm{J}$ - Trematopora maculata sp. nov., longitudinal section, holotype PMO 221.816. 

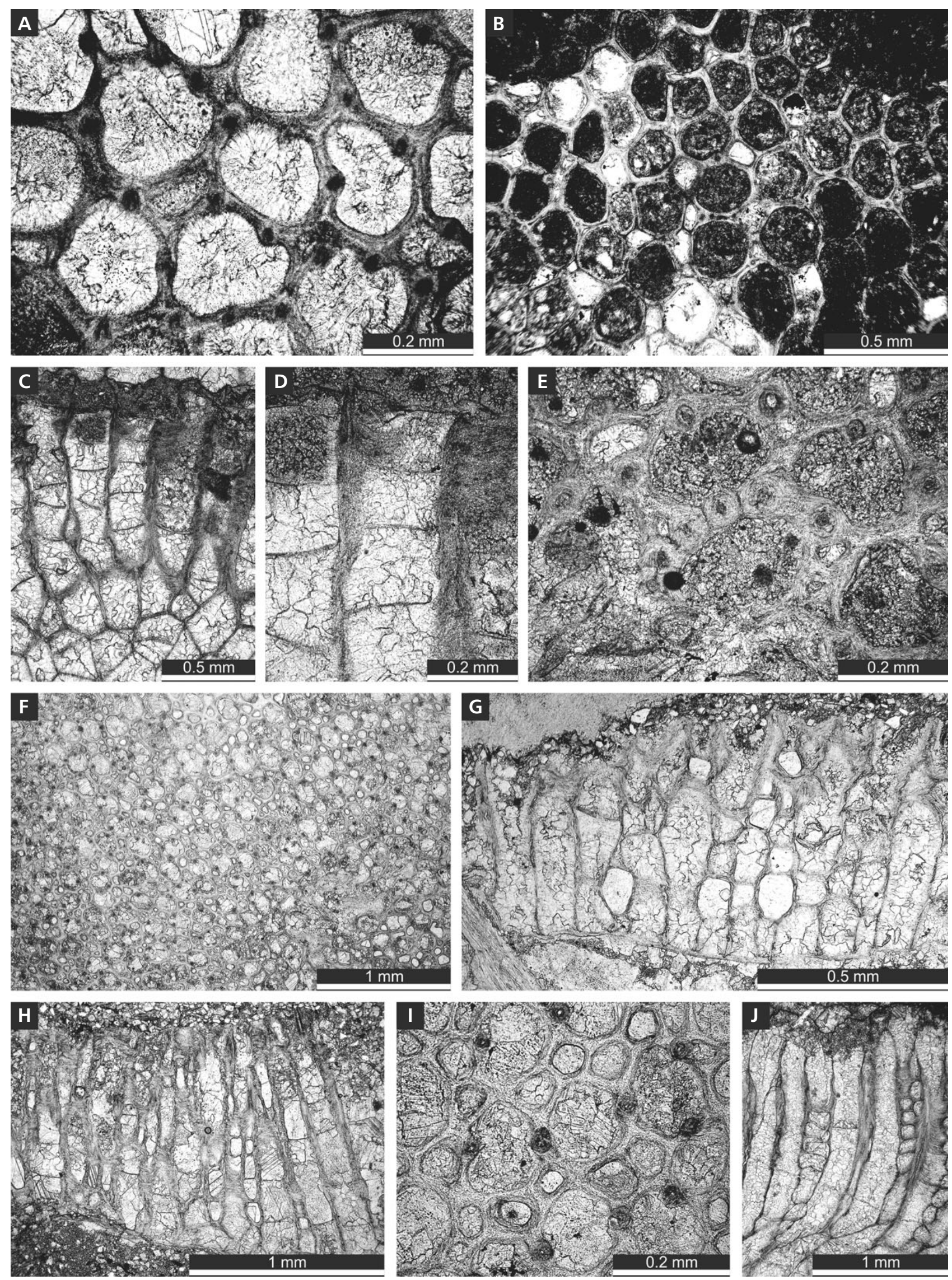
Remarks. - Trematopora maculata sp. nov. is similar to T. beikhemensis Astrova, 1959 from the Wenlock of western Tuva (Russia), but differs by presence of macrozooecia, broader exozone, and thicker walls. Trematopora maculata sp. nov. differs from T. cristata Kopaevich, 1984 from the Wenlock of Mongolia by presence of macrozooecia and less elongated apertures.

Occurrence. - Steinsfjorden Formation, Brattstad Member, Silurian, Wenlock, Sheinwoodian-Homerian; Ødegårdsviken, Ringerike, Norway.

Suborder Amplexoporina Astrova, 1965

Family Amplexoporidae Miller, 1889

\section{Genus Amplexopora Ulrich, 1882}

Type species. - Atactopora septosa Ulrich, 1879. Upper Ordovician; USA.

Diagnosis. - Ramose, frondescent, incrusting or massive colonies. Monticules generally well developed. Wall structure commonly appearing integrate in the exozone, sometimes amalgamate. Laminae from adjacent zooecia intersect a sharply defined zooecial boundary at angles of less than $90^{\circ}$ to form a V-shaped pattern pointing distally. Distinct zooecial linings present in several species. Diaphragms are extremely variable in thickness, curvature, parallelism and spacing, with compound and cystoidal diaphragms and cystiphragms common in many species. Mesozooecia lacking or few; early chambers are beaded and later diaphragms regularly and closely spaced. Acanthostyles usually abundant and of two types: those that are concentrated generally in zooecial and extend throughout the width of the exozone, occurring in all species; and additional acanthostyles that are concentrated between zooecial corners and extend through a part of the exozone width occurring in some species. These additional acanthostyles cause inflections of zooecial walls.

Remarks. - Amplexopora differs from Monotrypa by abundant diaphragms and wall microstructure with sharply defined zooecial boundary.

Boardman (1960) revised the genus Amplexopora and designated Atactopora septosa Ulrich, 1879 as the type species. According to the emended diagnosis of Boardman (1960) (followed here), Amplexopora possesses mesozooecia with abundant diaphragms. On the contrary, Astrova
(1978, p. 105) mentioned exilazooecia in Amplexopora, tube-like heterozooecia without or with few diaphragms. Based on this character she defined the suborder Amplexoporina, which comprises trepostome bryozoans with exilazooecia. Furthermore, Astrova (1978) re-designated Amplexopora cingulata Ulrich, 1882 as the type species of Amplexopora. According to Boardman (1960, p. 17) this species was never described or figured (except in Astrova 1978, pl. 17, fig. 2).

Occurrence. - Lower Ordovician-lower Silurian; North America, Australia, Siberia, Europe.

\section{Amplexopora crassiparietum sp. nov. Figure 9A-F, Table 6}

Etymology. - The species is named after its thick walls (from Latin "crassus" = thick, and "parietis" = wall). Holotype. - PMO 221.770.

Paratypes. - PMO 221.750-221.755, PMO 221.757, PMO 221.760, PMO 221.761-221.762, PMO 221.765-221.769, PMO 221.765, PMO 221.807-221.808, PMO 221.810, PMO 221.811, PMO 221.814.

Type locality. - Nes bus stop, Ringerike, Norway.

Type stratum. - Steinsfjorden Formation, Brattstad Member, Silurian, Wenlock, Sheinwoodian-Homerian.

Diagnosis. - Branched colonies with distinct wide exozones; autozooecial apertures polygonal; mesozooecia rare; acanthostyles common, spaced 1 to 5 around autozooecial apertures, moderate in size, locally absent; maculae consisting of macrozooecia.

Description. - Branched colonies, branch diameter 2.75-5.20 mm. Exozone distinct, 0.40-1.40 mm wide, endozone 1.55-2.90 mm wide. Secondary overgrowths absent. Autozooecia long, growing parallel to branch axis for a long distance in endozone, in exozone bending, having polygonal shape in transverse section in endozone. Autozooecial apertures polygonal. Diaphragms in endozone rare, planar, thin; in exozone abundant, straight or slightly curved. Mesozooecia rare, small, polygonal in cross section, restricted to exozone. Acanthostyles common, spaced 1 to 5 around autozooecial apertures, moderate in size, having distinct hyaline cores and wide,

Figure 8. Trematopora maculata sp. nov. • A-C - longitudinal section, holotype PMO 221.816. • D - tangential section, holotype PMO 221.816. - E - longitudinal section of secondary encrusting, paratype PMO 221.827. • F - longitudinal section, paratype PMO 221.776. $\bullet \mathrm{G}-$ transverse section, paratype PMO 221.817. $\bullet \mathrm{H}$ - tangential section, paratype PMO 221.824. 

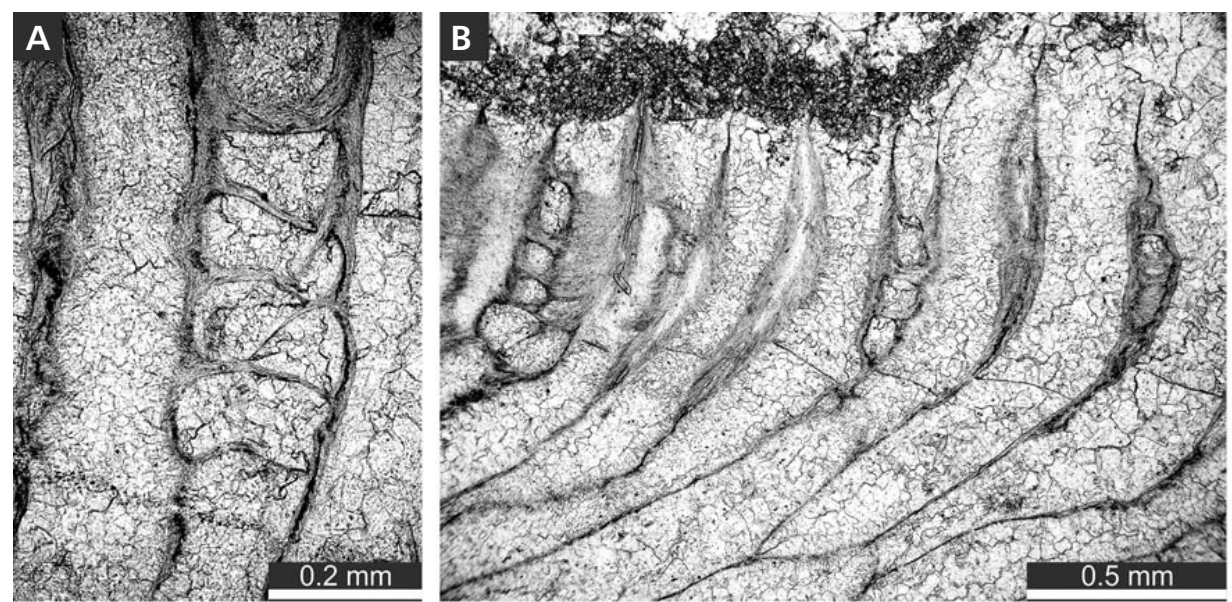
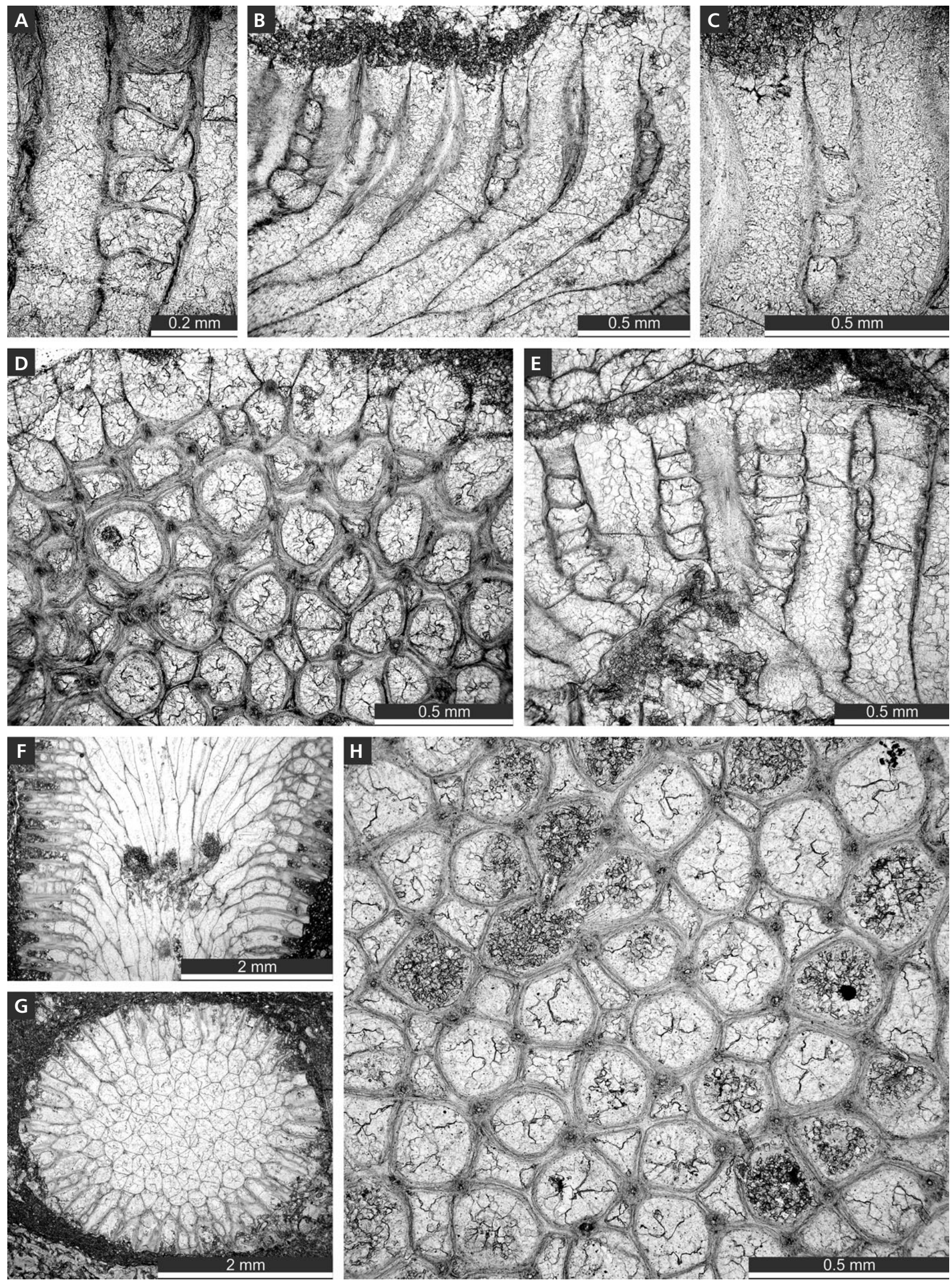
Table 6. Measurements of Amplexopora crassiparietum sp. nov. Abbreviations as in Table 1 .

\begin{tabular}{|c|c|c|c|c|c|c|}
\hline & $\mathrm{N}$ & $\mathrm{X}$ & $\mathrm{SD}$ & $\mathrm{CV}$ & MIN & MAX \\
\hline Branch width, mm & 10 & 3.91 & 0.916 & 23.43 & 2.75 & 5.20 \\
\hline Exozone width, mm & 10 & 0.90 & 0.312 & 34.64 & 0.40 & 1.40 \\
\hline Endozone width, mm & 10 & 2.11 & 0.439 & 20.80 & 1.55 & 2.90 \\
\hline Aperture width, mm & 40 & 0.11 & 0.017 & 15.93 & 0.08 & 0.14 \\
\hline Aperture spacing, $\mathrm{mm}$ & 40 & 0.20 & 0.023 & 11.33 & 0.15 & 0.26 \\
\hline $\begin{array}{l}\text { Aperture width, mm } \\
\text { (macular) }\end{array}$ & 16 & 0.19 & 0.030 & 15.72 & 0.15 & 0.25 \\
\hline $\begin{array}{l}\text { Aperture spacing, } \mathrm{mm} \\
\text { (macular) }\end{array}$ & 16 & 0.26 & 0.023 & 9.14 & 0.22 & 0.30 \\
\hline Mesozooecia width, mm & 30 & 0.048 & 0.013 & 26.91 & 0.025 & 0.078 \\
\hline Acanthostyle diameter, $\mathrm{mm}$ & 30 & 0.034 & 0.006 & 17.36 & 0.025 & 0.050 \\
\hline Acanthostyles per aperture & 40 & 3.1 & 0.888 & 28.89 & 1.0 & 5.0 \\
\hline $\begin{array}{l}\text { Autozooecial diaphragms } \\
\text { spacing, mm }\end{array}$ & 40 & 0.15 & 0.044 & 29.09 & 0.07 & 0.24 \\
\hline Exozonal wall thickness, mm & 40 & 0.079 & 0.023 & 29.50 & 0.030 & 0.135 \\
\hline
\end{tabular}

Table 7. Measurements of Amplexopora evae sp. nov. Abbreviations as in Table 1.

\begin{tabular}{|c|c|c|c|c|c|c|}
\hline & $\mathrm{N}$ & $\mathrm{X}$ & $\mathrm{SD}$ & $\mathrm{CV}$ & MIN & MAX \\
\hline anch width, mm & 6 & 3.10 & 0.334 & 10.78 & 2.55 & 3.40 \\
\hline Exozone width, mm & 6 & 0.74 & 0.309 & 41.65 & 0.35 & 1.00 \\
\hline Endozone width, mm & 6 & 1.62 & 0.386 & 23.82 & 1.10 & 2.18 \\
\hline Aperture width, mm & 85 & 0.15 & 0.026 & 17.02 & 0.10 & 0.21 \\
\hline Ap & 85 & 0.19 & 0.031 & 15.82 & 0.12 & 0.30 \\
\hline $\begin{array}{l}\text { Aperture width, mm } \\
\text { (macular) }\end{array}$ & 16 & 0.20 & 0.015 & 7.35 & 0.18 & 0.23 \\
\hline $\begin{array}{l}\text { Aperture spacing, mm } \\
\text { (macular) }\end{array}$ & 16 & 0.27 & 0.039 & 14.57 & 0.20 & 0.33 \\
\hline Acanthostyle diameter, mm & 61 & 0.049 & 0.013 & 26.40 & 0.025 & 0.080 \\
\hline Acanthostyles per aperture & 75 & 2.7 & 1.075 & 39.72 & 1.0 & 5.0 \\
\hline Mesozooecia width, mm & 60 & 0.05 & 0.018 & 36.31 & 0.02 & 0.09 \\
\hline $\begin{array}{l}\text { Mesozooecial diaphragms } \\
\text { spacing, mm }\end{array}$ & 20 & 0.12 & 0.036 & 30.16 & 0.07 & 0.20 \\
\hline $\begin{array}{l}\text { Autozooecial diaphragms } \\
\text { spacing, mm }\end{array}$ & 49 & 0.19 & 0.061 & 31.70 & 0.09 & 0.35 \\
\hline Exozonal wall thickness, $\mathrm{mm}$ & 40 & 0.046 & 0.013 & 29.05 & 0.025 & 0.075 \\
\hline
\end{tabular}

dark sheaths, situated in the dark median lining of autozooecial walls, originating from base of exozone; locally acanthostyles absent. Autozooecial walls in endozone $0.005-0.010 \mathrm{~mm}$ thick, granular; in exozone 0.030 to $0.135 \mathrm{~mm}$ thick, displaying reverse $\mathrm{V}$-shaped lamination with dark, serrated median lining. Maculae indistinct, $0.8-1.3 \mathrm{~mm}$ in diameter, consisting of macrozooecia.
Remarks. - Amplexopora crassiparietum sp. nov. differs from A. silurica Astrova, 1970 from the lower Silurian (Llandovery) of Ukraine (Podolia) in smaller autozooecial apertures (aperture width $0.08-0.14 \mathrm{~mm}$ vs $0.17-0.20 \mathrm{~mm}$ in A. silurica), and in less abundant acanthostyles (1-5 vs 4-7 per autozooecial aperture in A. silurica).

Occurrence. - Steinsfjorden Formation, Brattstad Member, Silurian, Wenlock, Sheinwoodian-Homerian; Nes bus stop and Åsa, Ringerike, Norway.

\section{Amplexopora evae sp. nov.}

Figures 10A-F, 11A, B, Table 7

Etymology. - The species is named for the junior author's wife to honour her participation in bryozoan fieldwork.

Holotype. - PMO 170.890.

Paratypes. - PMO 170.886, PMO 170.882, PMO 170.887-170.888, PMO 221.771-221.772, PMO 221.773-221.777, PMO 221.780, PMO 221.804-221.805.

Type locality. - Ødegårdsviken, Ringerike, Norway.

Type stratum. - Steinsfjorden Formation, Brattstad Member, Silurian, Wenlock, Sheinwoodian-Homerian.

Diagnosis. - Branched and encrusting colonies; branches with distinct wide exozones; autozooecial apertures polygonal; mesozooecia rare; acanthostyles common, spaced 1 to 5 around autozooecial apertures, moderate in size, locally absent; maculae consisting of macrozooecia.

Description. - Branched colonies, branch diameter 2.55-3.40 $\mathrm{mm}$. Exozone distinct, 0.35-1.00 mm wide, endozone 1.10-2.18 mm wide. Secondary overgrowths common, 0.65-2.50 mm thick. Encrusting colonies 1.5-2.5 mm thick. In branched colonies, autozooecia long, growing parallel to branch axis for a long distance in endozone, in exozone bending, having polygonal shape in transverse section in endozone. In encrusting colonies autozooecia arise directly from the epitheca with narrow endozonal part. Autozooecial apertures polygonal. Diaphragms in endozone rare, planar, thin; in exozone abundant, straight or slightly curved. Mesozooecia rare, small, polygonal in cross section, restricted to exozone. Acanthostyles common, spaced 1 to 5 around autozooecial apertures, moderate in size, having distinct hyaline cores and wide, dark

Figure 9. Amplexopora crassiparietum sp. nov. • A - longitudinal section, holotype PMO 221770. • B, C - transverse section, holotype PMO 221.770. • D - longitudinal section, holotype PMO 221.770. • E - tangential section, holotype PMO 221.770. $\bullet \mathrm{F}-$ tangential section, paratype PMO 221.760. 

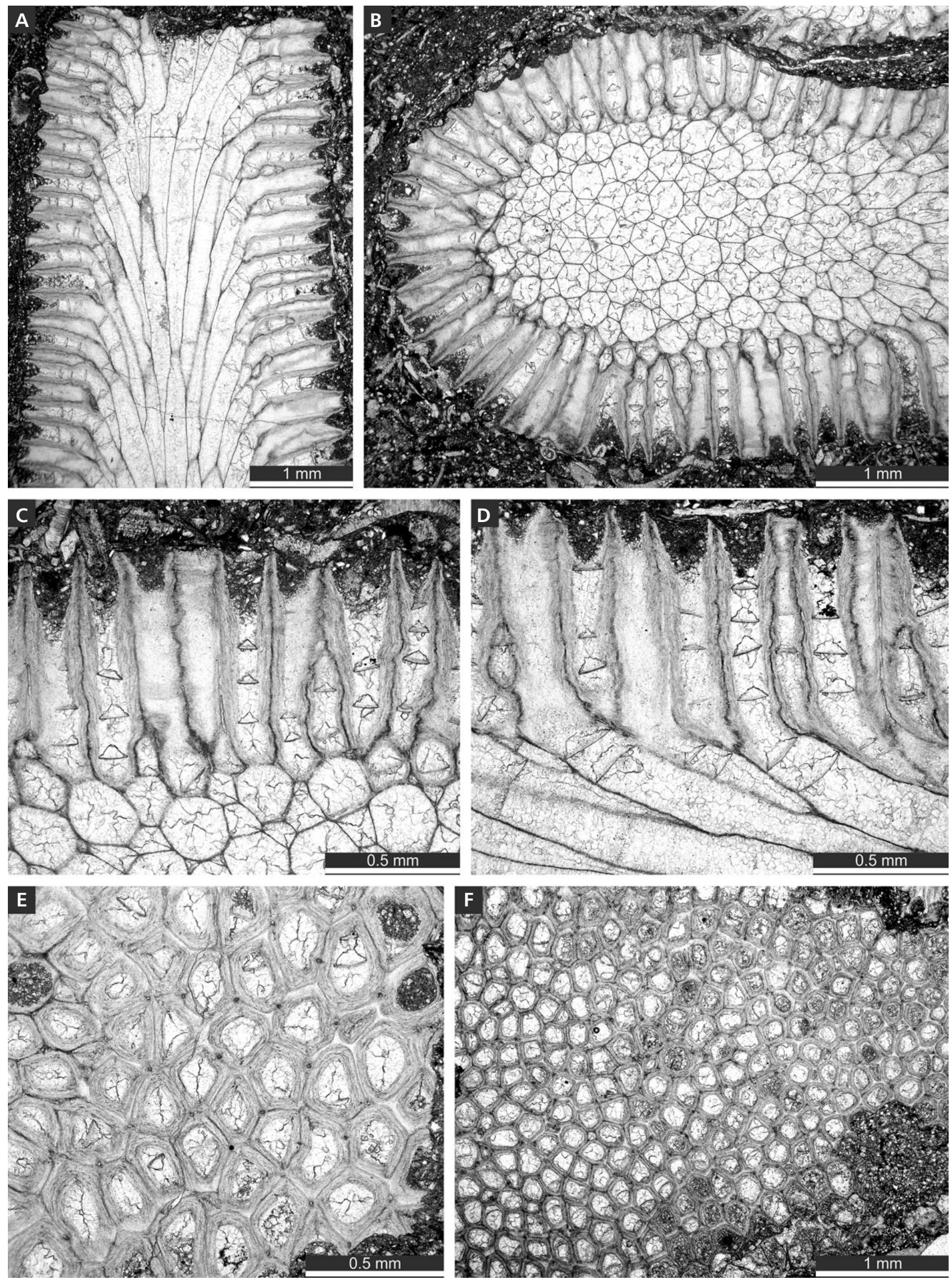
Table 8. Measurements of Eridotrypella sp. Abbreviations as in Table 1 .

\begin{tabular}{lcccccc}
\hline & $\mathrm{N}$ & $\mathrm{X}$ & $\mathrm{SD}$ & $\mathrm{CV}$ & $\mathrm{MIN}$ & $\mathrm{MAX}$ \\
\hline Aperture width, mm & 20 & 0.11 & 0.013 & 11.15 & 0.08 & 0.13 \\
Aperture spacing, mm & 20 & 0.19 & 0.022 & 11.51 & 0.16 & 0.22 \\
Exilazooecia width, mm & 7 & 0.05 & 0.014 & 29.91 & 0.03 & 0.07 \\
\hline
\end{tabular}

sheaths, situated in the dark median lining of autozooecial walls, originating from base of exozone; locally acanthostyles absent. Autozooecial walls in endozone 0.005-0.010 mm thick, granular; in exozone $0.025-0.075 \mathrm{~mm}$ thick, displaying reverse $\mathrm{V}$-shaped lamination with dark, serrated median lining. Maculae indistinct, consisting of macrozooecia, $0.70-1.35 \mathrm{~mm}$ in diameter.

Remarks. - Amplexopora evae sp. nov. differs from A. crassiparietum sp. nov. in thinner autozooecial walls and larger autozooecial apertures.

Occurrence. - Steinsfjorden Formation, Brattstad Member, Silurian, Wenlock, Sheinwoodian-Homerian; Ødegårdsviken, Ringerike, Norway.

Family Eridotrypellidae Morozova, 1961

\section{Genus Eridotrypella Duncan, 1939}

Type species. - Batostomella obliqua Ulrich, 1890. Middle Devonian; Michigan (USA).

Diagnosis. - Branched colonies. Autozooecial apertures irregularly polygonal. Autozooecial walls laminated, without distinct zooecial boundaries, irregularly thickened, containing spherulites. Diaphragms complete, varying in number. Exilazooecia rare. Acanthostyles varying in size and number.

Comparison. - Eridotrypella Duncan, 1939 differs from Eostenopora Duncan, 1939 in colony form (ramose branched $v s$ encrusting or massive colonies).

Occurrence. - Silurian-?Carboniferous; worldwide.

\section{Eridotrypella sp. A}

Figure $11 \mathrm{C}-\mathrm{H}$, Table 8

Material. - PMO 221.789, PMO 221.782, PMO 221.796.
Description. - Ramose branched colonies, $1.2 \mathrm{~mm}$ in diameter. Exozone $0.34 \mathrm{~mm}$ wide, endozone $0.52 \mathrm{~mm}$ wide. Exozones distinctly separated from endozones. Secondary overgrowth occurring, 0.28-0.66 mm thick. Autozooecia long in endozones, bending sharply in exozones. Autozooecial apertures polygonal with rounded corners. Autozooecial diaphragms absent to rare in endozones; common to abundant in transition between endozone and exozone, straight or inclined. Exilazooecia rare, short, polygonal in cross section. Acanthostyles rare, having distinct narrow cores and laminated sheaths, $0.030-0.035 \mathrm{~mm}$ in diameter. Autozooecial walls granular, locally weakly crenulated, $0.005-0.010 \mathrm{~mm}$ thick in endozones; serrated in the longitudinal view and merged in the tangential section, 0.04-0.06 mm thick in exozones. Maculae not observed.

Remarks. - Eridotrypella sp. differs from E. sepizensis Astrova, 1970 from the lower Silurian (Wenlock) of Estonia in less abundant acanthostyles and in larger apertures (aperture width 0.08-0.13 $\mathrm{mm}$ vs 0.13-0.20 mm in E. sepizensis). Eridotrypella sp. differs from E. duncanae Pushkin, 1976 from the upper Silurian (Ludlow) of Belarus in narrower branches (branch width $1.2 \mathrm{~mm} v s$ 2.5-5.0 $\mathrm{mm}$ in E. duncanae), and in smaller apertures (aperture width 0.08-0.13 mm vs $0.14-0.25 \mathrm{~mm}$ in E. duncanae).

Occurrence. - Steinsfjorden Formation, Brattstad Member, Silurian, Wenlock, Sheinwoodian-Homerian; Ødegårdsviken, Ringerike, Norway.

Order Cryptostomata Vine, 1884

Suborder Rhabdomesina Astrova \& Morozova, 1956

Family Rhabdomesidae Vine, 1884

\section{Genus Orthopora Hall, 1886}

Type species. - Trematopora regularis Hall, 1874. Lower Devonian; USA.

Diagnosis. - Branched colonies. Autozooecia short, budding from more or less distinct medial axis in spiral order. Autozooecial diaphragms rare to absent. Both superior and inferior hemisepta commonly present; sometimes double hemisepta occurring; rarely hemisepta absent. Autozooecial apertures oval, arranged regularly in alternating rows on the colony surface. Walls granular in the endozone; laminated in exozone. Paurostyles abundant, prominent. Acanthostyles present, less abundant than paurostyles. Heterozooecia absent.

Figure 10. Amplexopora evae sp. nov., holotype PMO $170.890 \cdot \bullet A-$ general view of a branched colony. $\bullet \mathrm{B}-$ transverse section. $\bullet \mathrm{C}-$ longitudinal section. $\bullet \mathrm{D}$ - longitudinal section of encrusting colony. $\bullet \mathrm{E}$ - transverse section showing wall structure. $\bullet \mathrm{F}-$ tangential section. 

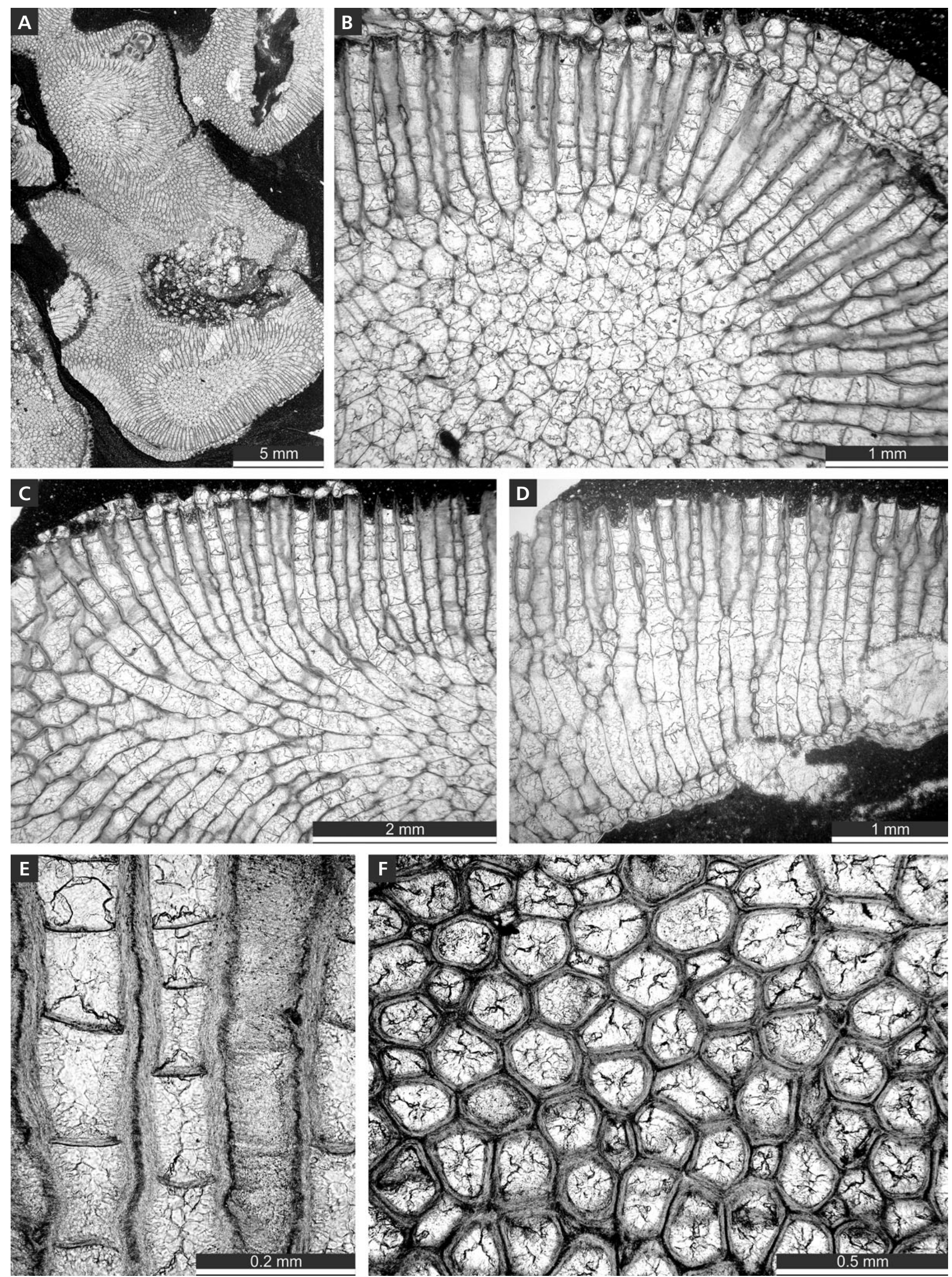
Table 9. Measurements of Orthopora worsleyi sp. nov. Abbreviations as in Table 1 .

\begin{tabular}{lcccccc}
\hline & $\mathrm{N}$ & $\mathrm{X}$ & $\mathrm{SD}$ & $\mathrm{CV}$ & $\mathrm{MIN}$ & $\mathrm{MAX}$ \\
\hline Branch width, mm & 21 & 0.59 & 0.098 & 16.57 & 0.42 & 0.77 \\
Exozone width, mm & 21 & 0.17 & 0.041 & 24.03 & 0.10 & 0.25 \\
Endozone width, mm & 21 & 0.25 & 0.064 & 25.33 & 0.13 & 0.35 \\
$\begin{array}{l}\text { Aperture width, mm } \\
\begin{array}{l}\text { Aperture spacing along branch, } \\
\text { mm }\end{array}\end{array}$ & 25 & 0.06 & 0.012 & 19.06 & 0.04 & 0.08 \\
$\begin{array}{l}\text { Aperture spacing diagonally, } \\
\text { mm }\end{array}$ & 25 & 0.15 & 0.019 & 12.57 & 0.12 & 0.20 \\
\begin{tabular}{l} 
Acanthostyle diameter, mm \\
\hline
\end{tabular} & 30 & 0.031 & 0.006 & 18.38 & 0.025 & 0.045 \\
\hline
\end{tabular}

Table 10. Measurements of Mediaporina kiaeri sp. nov. Abbreviations as in Table 1 .

\begin{tabular}{lcccccc}
\hline & $\mathrm{N}$ & $\mathrm{X}$ & $\mathrm{SD}$ & $\mathrm{CV}$ & $\mathrm{MIN}$ & $\mathrm{MAX}$ \\
\hline Aperture width, mm & 19 & 0.08 & 0.014 & 17.79 & 0.06 & 0.10 \\
Aperture spacing, mm & 20 & 0.17 & 0.017 & 9.59 & 0.14 & 0.2 \\
$\begin{array}{l}\text { Heterozooecia width, mm } \\
\begin{array}{l}\text { Acanthostyle diameter, } \\
\text { mm }\end{array}\end{array}$ & 20 & 0.03 & 0.008 & 28.09 & 0.015 & 0.045 \\
\hline
\end{tabular}

Remarks. - Orthopora Hall, 1886 differs from Trematella Hall, 1886 in absence of metazooecia and in presence of well-developed hemisepta.

Occurrence. - Silurian to Carboniferous of North America, Europe and China, Middle Permian of Oman.

Orthopora worsleyi sp. nov.

Figure 13A-H, Table 9

Etymology. - The species is named for David Worsley, who has been an inspiration to us in the study of Silurian rocks of the Oslo Region.

Holotype. - PMO 221.790.

Paratypes. - PMO 221.750-221.755, PMO 221.757, PMO 221.781-221.782, PMO 221.784-221.785, PMO 221.789, PMO 221.794, PMO 221.796.

Type locality. - Nes bus stop, Ringerike Norway.

Type stratum. - Steinsfjorden Formation, Brattstad Member, Silurian, Wenlock, Sheinwoodian-Homerian.
Diagnosis. - Branched colonies; bifurcation common; both superior and inferior hemisepta well developed; acanthostyles abundant, arranged in single row between autozooecia, varying in size; paurostyles abundant.

Description. - Branched colonies arising from encrusting bases. Branches $0.42-0.77 \mathrm{~mm}$ in diameter, with $0.13-0.35 \mathrm{~mm}$ wide endozones and $0.10-0.25 \mathrm{~mm}$ wide exozones. Branch bifurcation common. Transverse sections of branches circular. Autozooecia medium in length, budding from indistinct medial axis in spiral order. Autozooecial diaphragms rare to absent. Superior hemisepta present, blunt, curved proximally, positioned near the bend of autozooecial chamber; inferior hemisepta long, positioned beneath superior hemisepta, curved distally. Autozooecial apertures oval, arranged regularly in alternating rows on the colony surface. Walls in the endozone granular, 0.003-0.005 mm thick; laminated in exozone. Acanthostyles abundant, arranged in longitudinal rows between apertures, varying in size, having narrow hyaline cores and wide laminated sheaths. Paurostyles abundant, arranged irregularly between acanthostyles, $0.008-0.016 \mathrm{~mm}$ in diameter. Heterozooecia absent.

Remarks. - Orthopora worsleyi sp. nov. differs from O. casualis Goryunova, 1985 from the lower Silurian Rochester Shale (Wenlock) of New York, USA in narrower branches $(0.42-0.77 \mathrm{~mm}$ vs $0.80-1.20 \mathrm{~mm}$ in $O$. casualis), and in slightly smaller apertures (aperture width 0.04-0.08 $\mathrm{mm}$ vs $0.05-0.10 \mathrm{~mm}$ in O. casualis). Orthopora worsleyi sp. nov. differs from species described by Kopaevich (1975) as Orthopora rhombifera (Hall, 1874) from the upper Silurian (Př́idolí) of Estonia in narrower branches $(0.42-0.77 \mathrm{~mm}$ vs $0.76-1.17 \mathrm{~mm}$ in that species).

Occurrence. - Steinsfjorden Formation, Brattstad Member, Silurian, Wenlock, Sheinwoodian-Homerian; Ødegårdsviken, Ringerike, Norway.

Family Arthrostylidae Ulrich, 1882

\section{Mediaporina Pushkin in Pushkin et al., 1990}

Type species. - Mediapora aspersa Kopaevich, 1975. Upper Silurian, Ludlow; Estonia.

Diagnosis. - Branched colonies. Autozooecia relatively

Figure 11. A, B -Amplexopora evae sp. nov., tangential section, holotype PMO 170.890 . $\bullet \mathrm{C}-\mathrm{H}-$ Eridotrypella sp.; C, D - transverse section, paratype PMO 221.782. E - longitudinal section, holotype PMO 221.789. $\bullet \mathrm{F}, \mathrm{G}-$ tangential section, paratype PMO 221.789 . $\bullet$ H - longitudinal section, holotype PMO 221.789. 

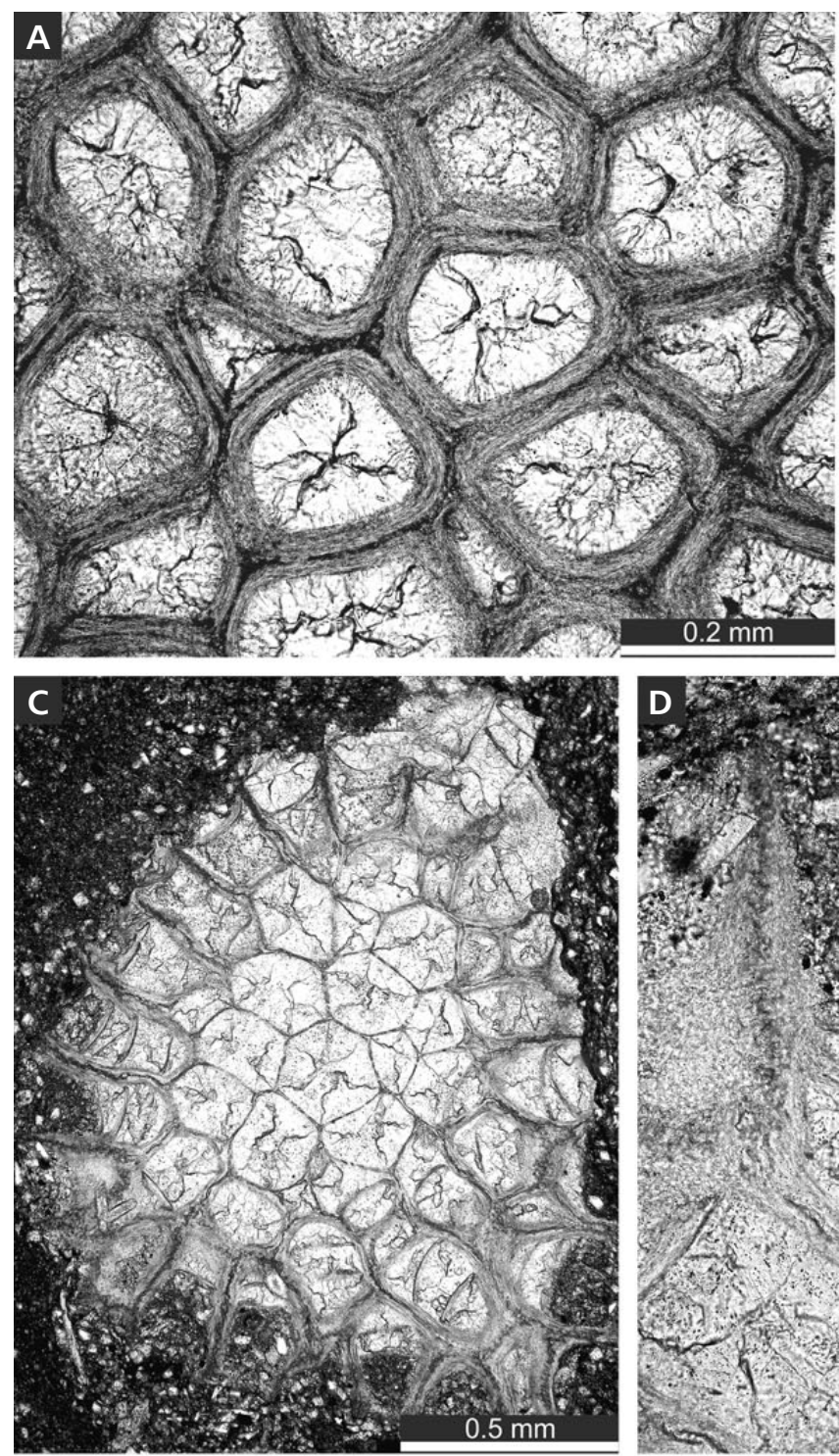
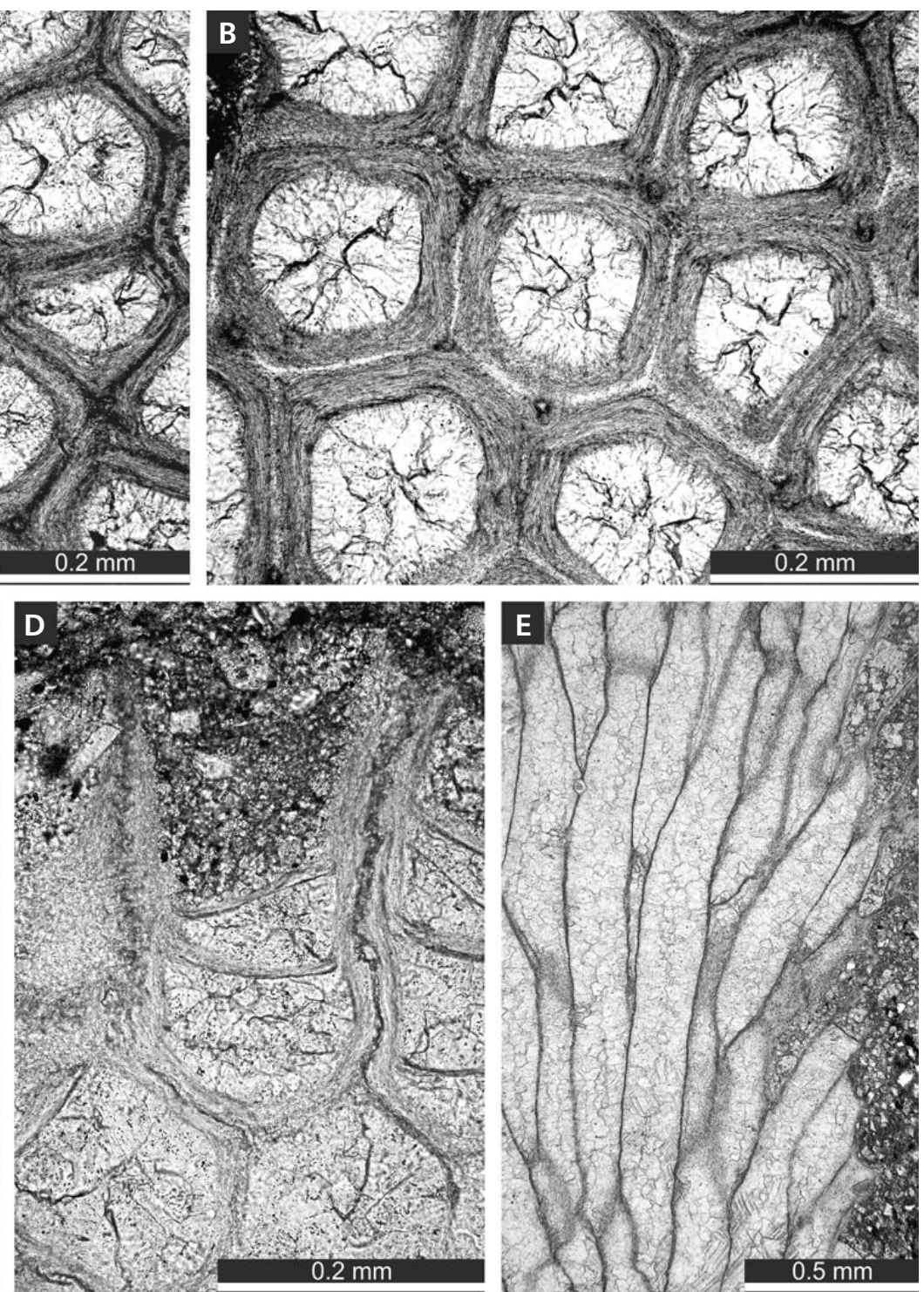
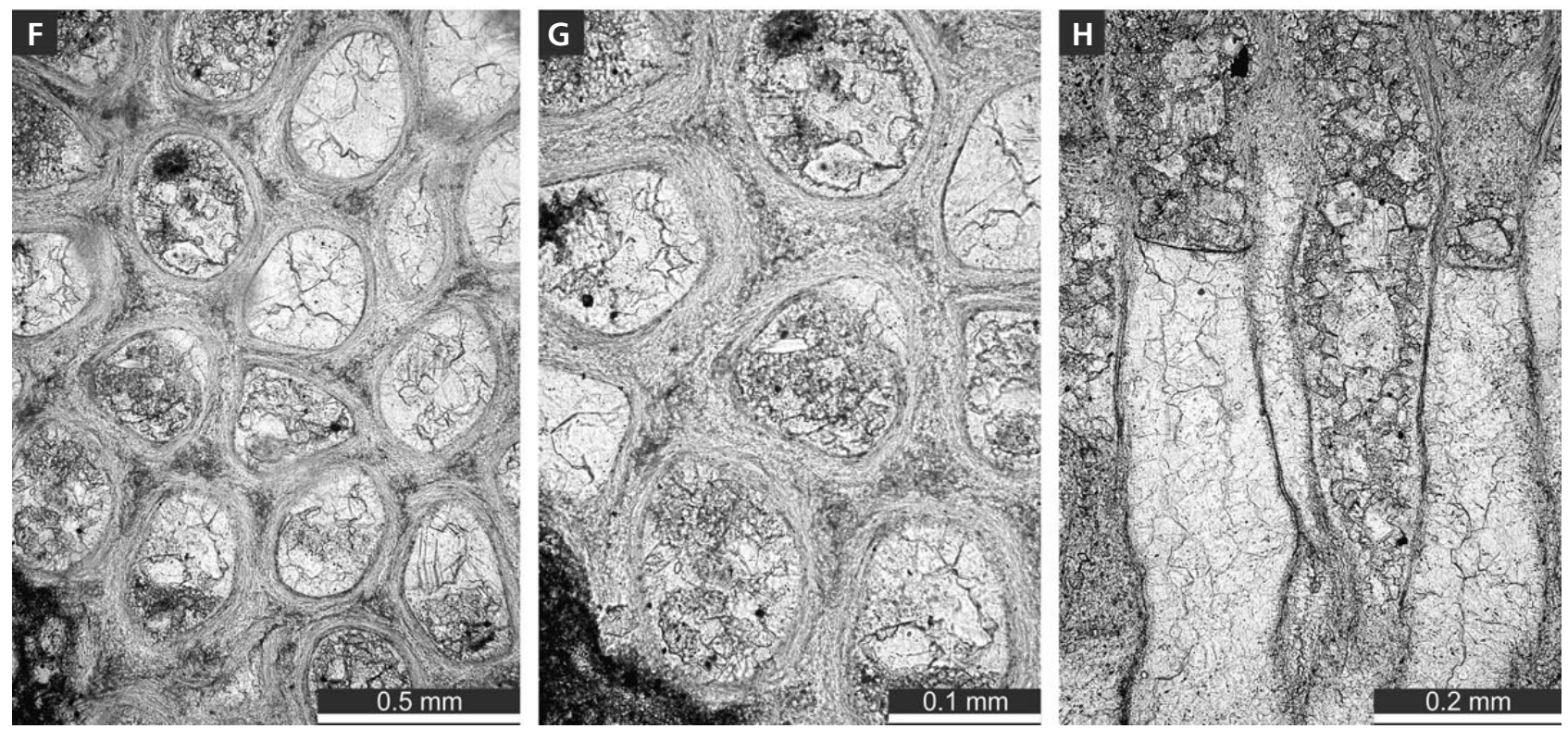

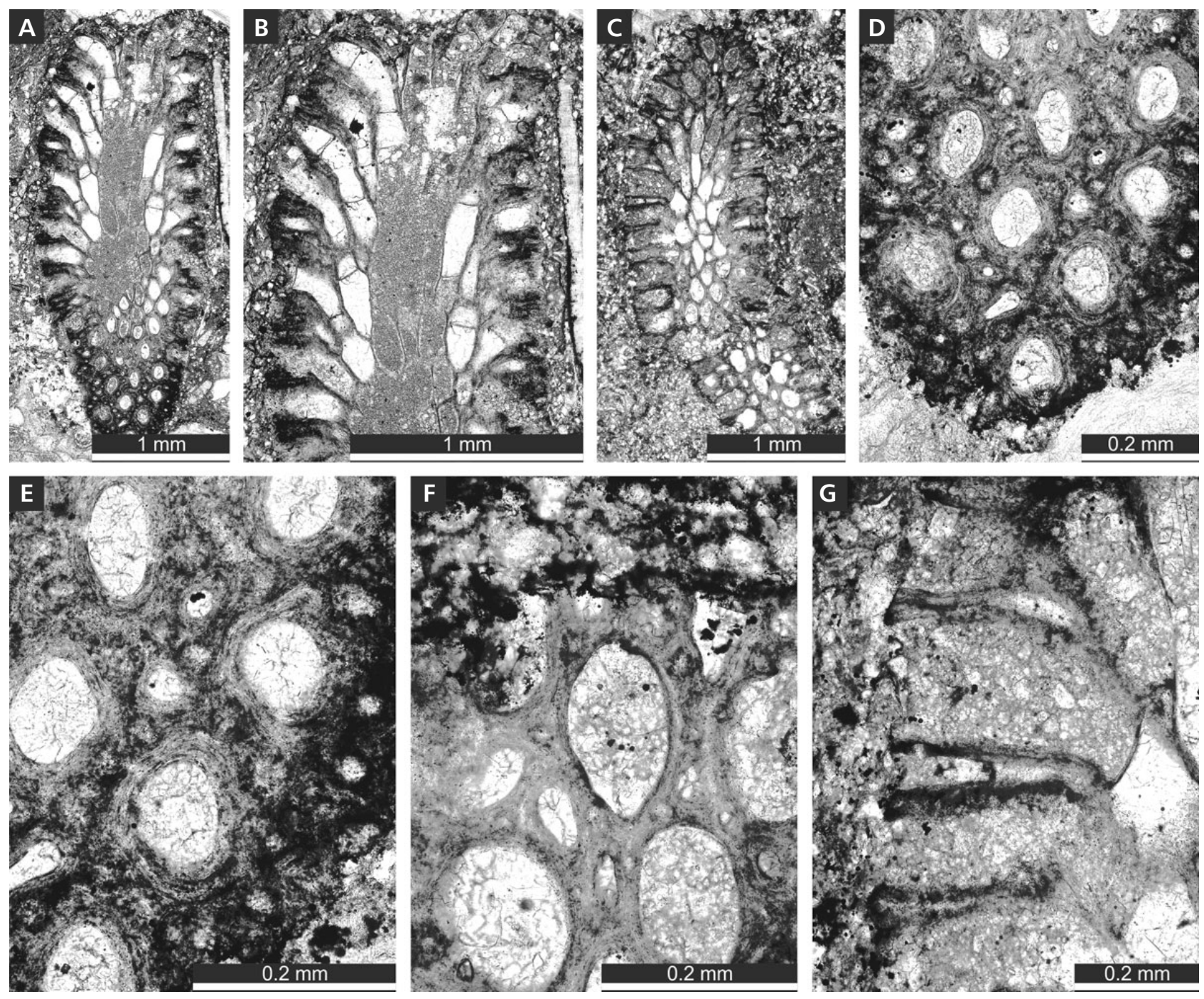

Figure 12. Mediaporina kiaeri sp. nov. $\bullet$ A, B - longitudinal section, holotype PMO 221.754. $\bullet$ C - oblique section, paratype PMO 221.762. • D, E - tangential section showing autozooecial apertures, styles and heterozooecia, holotype PMO 221.754. $\bullet \mathrm{F}$ - deep tangential section showing autozooecial apertures and heterozooecia, paratype PMO 221.762. • G - longitudinal section showing autozooecia and heterozooecia, paratype PMO 221.762.

short, budding from more or less distinct medial axis in spiral order. Autozooecial diaphragms rare to common. Hemisepta absent. Autozooecial apertures oval, arranged regularly in alternating rows on the colony surface. Walls granular in the endozone; laminated in exozone. Paurostyles large, abundant, prominent. Acanthostyles absent. Heterozooecia tubular, common to abundant, restricted to exozone, bearing few diaphragms.

Remarks. - Mediaporina Pushkin in Pushkin et al., 1990 differs from Sceptropora Ulrich, 1888 in having branched colony instead of branched segments with expanded distal parts.

Occurrence. - Lower to upper Silurian of Europe (British Isles, Norway, Estonia, Belarus, Latvia).

\section{Mediaporina kiaeri sp. nov.}

Figure $12 \mathrm{~A}-\mathrm{G}$, Table 10

Etymology. - The species name refers to Johan A. Kiær

Figure 13. Orthopora worsleyi sp. nov. • A, B - oblique section, holotype PMO 221.790. • C, D - oblique section of the basal part of the colony, paratype PMO 221.794. $\bullet$ E - oblique section of dichotomous colony, PMO 221.783. • F, G - longitudinal section, PMO 221.784. $\bullet$ H - branch transverse section, paratype PMO 221.783. 

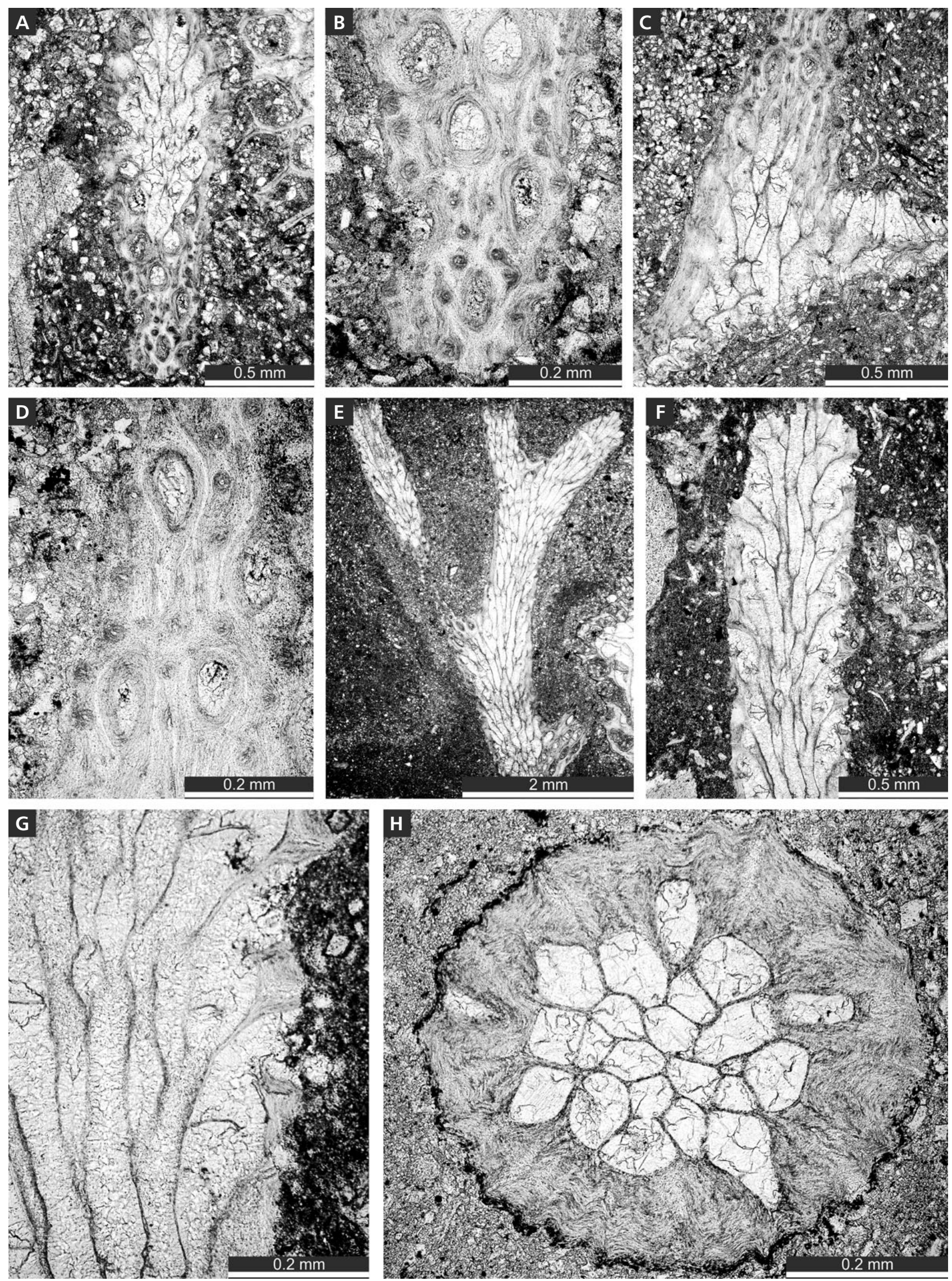
who described the Silurian rocks of Ringerike in great detail in 1908.

Holotype. - PMO 221.754.

Paratype. - PMO 221.762.

Type locality. - Nes bus stop, Ringerike Norway.

Type stratum. - Steinsfjorden Formation, Brattstad Member, Silurian, Wenlock, Sheinwoodian-Homerian.

Diagnosis. - Branched colonies; autozooecial diaphragms rare to common; acanthostyles abundant, 7-9 surrounding each autozooecia aperture; heterozooecia abundant, 4-6 surrounding each autozooecia aperture.

Description. - Branches 1.05-1.20 mm in diameter, with $0.30-0.37 \mathrm{~mm}$ wide endozones and $0.34-0.45 \mathrm{~mm}$ wide exozones. Branch bifurcation not observed. Autozooecia moderately long, growing along the branch axis in endozone and bending sharply in exozone, polygonal in transverse section of endozone. Autozooecial diaphragms rare to common, 2-3 usually present in each autozooecium. Hemisepta absent. Autozooecial apertures oval to slightly angular, arranged regularly in alternating rows on the colony surface. Walls in the endozone granular, 0.003-0.005 mm thick; indistinctly laminated in exozone. Acanthostyles abundant, 7-9 surrounding each autozooecia aperture, restricted to exozone, varying in size, having indistinct wide cores and narrow sheaths. Heterozooecia abundant, 4-6 surrounding each autozooecia aperture, originating at the base of exozone, rounded to oval at colony surface, containing thin diaphragms, often sealed by calcitic material at colony surface.

Remarks. - Mediaporina kiaeri sp. nov. differs from M. aspersa (Kopaevich, 1975) from the upper Silurian of Estonia by more abundant and smaller heterozooecia (heterozooecia width $0.015-0.045 \mathrm{~mm}$ vs $0.05-0.13 \mathrm{~mm}$ in $M$. aspersa) and by larger styles (style diameter $0.025-0.050 \mathrm{~mm} \mathrm{vs}$ 0.02-0.04 mm in M. aspersa). Mediaporina kiaeri sp. nov. differs from M. orbiculata (Pushkin, 1975) from the upper Silurian of Belarus in smaller autozooecial apertures (aperture width 0.06-0.10 $\mathrm{mm}$ vs $0.09-0.12 \mathrm{~mm}$ in $M$. orbiculata) and by more abundant heterozooecia.

Occurrence. - Steinsfjorden Formation, Brattstad Member, Silurian, Wenlock, Sheinwoodian-Homerian; Nes bus stop, Ringerike, Norway.

\section{Acknowledgements}

The Deutsche Akademische Austauschdienst (DAAD) supported Andrej Ernst's study visit to the Natural History Museum, Uni- versity of Oslo, with a one-year fellowship 2002-2003 (grant D/02/00949). Patrick N. Wyse Jackson, Dublin, and Caroline Buttler, Cardiff, are thanked for their helpful reviews.

\section{References}

ANStey, R.L. \& PerRy, T.G. 1970. Biometric procedures in taxonomic studies of Paleozoic bryozoans. Journal of Paleontology 44, 383-398.

Astrova, G.G. 1957. Some new species of bryozoans from the Silurian of Tuva. Materiali k osnovam paleontologii Akademii nauk SSSR 1, 5-14. [in Russian]

Astrova, G.G. 1959. Silurian bryozoans of the central and western Tuva. Trudy Paleontologischeskogo instituta Akademii nauk SSSR 79, 1-74. [in Russian]

Astrova, G.G. 1964. New Order of the Paleozoic Bryozoa. Paleontologicheskii zhurnal 1964(2), 22-31. [in Russian]

Astrova, G.G. 1965. Morphology, history of development and systematics of the Ordovician and Silurian Bryozoa. Trudy Paleontologicheskogo instituta Akademii nauk SSSR 106, 1-432. [in Russian]

Astrova, G.G. 1970. New Silurian and Early Devonian bryozoans of Orders Cystoporata and Trepostomata from Estonia and Podolia, 7-22. In Astrova, G.G. \& Chudinova, I.I. (eds) New species of Palaeozoic bryozoans and corals. Nauka, Moscow.

Astrova, G.G. 1978. The history of development, system, and phylogeny of the Bryozoa: Order Trepostomata. Trudy Paleontologicheskogo instituta Akademii nauk SSSR 169, 1-240. [in Russian]

Astrova, G.G. \& Morozova, I.P. 1956. On systematics of the order Cryptostomata. Doklady Akademii nauk SSSR 110(4), 661-664. [in Russian]

BASSLER, R.S. 1906. The bryozoan fauna of the Rochester Shale. U.S. Geological Survey Bulletin 292, 1-137.

BoARDMAN, R.S. 1960. A revision of the Ordovician bryozoan genera Batostoma, Anaphragma, and Amplexopora. Smithsonian Miscellaneous Collections 140(5), 1-28.

BorG, F. 1926. Studies on Recent cyclostomatous Bryozoa. Zoologiska Bidrag från Uppsala 10, 181-507.

Duncan, H. 1939. Trepostomoatous Bryozoa from the Traverse Group of Michigan. University of Michigan Paleontology Contributions 5(10), 171-270.

Ehrenberg, C.G. 1831. Symbolae Physicae, seu Icones et descptiones Corporum Naturalium novorum aut minus cognitorum, quae ex itineribus per Libyam, Aegiptum, Nubiam, Dongalaam, Syriam, Arabiam et Habessiniam, studia annis 1820-25, redirent. Pars Zoologica, 4, Animalia Evertebrata exclusis Insectis. 10 pls. Berolini.

Goryunova, R.V. 1985. Morphology, system und phylogeny of Bryozoa (Order Rhabdomesida). Trudy Paleontologicheskogo instituta Akademii nauk SSSR 208, 1-152. [in Russian]

Hageman, S.J. 1993. Effects of nonnormality on studies of the morphological variation of a rhabdomesine bryozoan, Streblotrypa (Streblascopora) prisca (Gabb \& Horn). The University of Kansas Paleontological Contributions 4, 1-13. 
HaLl, J. 1852. Organic remains of the lower middle division of the New York system. Natural History of New York. Part 6. Palaeontology of New York 2, 40-52, 144-173.

HaLl, J. 1874. Descriptions of Bryozoa and corals of the lower Heldelberg Group. Twenty-sixth annual report of the New York State Museum of Natural History, 93-115.

Hall, J. 1886. Bryozoa of the Upper Helderberg Group; plates and explanations. State Geologist of New York, Annual Report for 1885, 25-53.

HenNig, A. 1908. Gotlands Silurbryozoen 3. Arkiv för zoologi 4, $1-64$.

Hetherington, C.J., Nakrem, H.A. \& Potel, S. 2011. Note on the composition and mineralogy of Wenlock Silurian bentonites from the Ringerike District: Implications for local and regional stratigraphic correlation and sedimentary environments. Norwegian Journal of Geology 91, 181-192.

KIÆR, J. 1908. Das Obersilur im Kristianiagebiete. Eine stratigraphisch-faunistische Untersuchung. Skrifter udgivne af Videnskabs-selskabet i Christiania. 595 pp. J. Dybwad, Christiania.

Kopaevich, G.V. 1975. Silurian Bryozoa of Estonia and Podoloia (Cryptostomata and Rhabdomesonata). Trudy Paleontologischeskogo instituta Akademii nauk SSSR 151, 5-153. [in Russian]

KopaEvich, G.V. 1984. Atlas of Bryozoa from the Ordovician, Silurian and Devonian of Mongolia. Sovmestnaya sovetskomongolskaya paleontologischeskaya ekspeditsiya, Trudy 22, $1-164$. [in Russian]

M'Coy, F. 1849. On some new genera and species of Palaeozoic corals and Foraminifera. Annals and Magazine of Natural History 3(2), 119-136. DOI 10.1080/03745485909494606

Melchin, M.J., SAdler, P.M. \& CRAmer, B.D. 2012. Chapter 21: The Silurian Period, 525-558. In Gradstein, F.M., OGG, J.G., Schmitz, M. \& OGG, G. (eds) The Geologic Time Scale 2012. Elsevier, New York.

Miller, S.A. 1889. North American Geology and Paleontology for the use of amateurs, students, and scientists. 664 pp. Western Methodist Book concern, Cincinnati.

Milne-Edwards, H. \& Haime, J. 1851. Monographie des polypiers fossiles des terrains paléozoïques, précédé d'un tableau général de la classification des Polypes. Archives du Muséum d'histoire naturelle 5, 1-504.

Morozova, I.P. 1961. Devonian Bryozoa of the Minusinsk and Kuznetsk basins. Trudy Paleontologischeskogo instituta Akademii nauk SSSR 86, 1-207. [in Russian]

Nicholson, H.A. 1879. On the structure and affinities of the "Tabulate Corals" of the Paleozoic period, with critical descriptions of illustrative species. $342 \mathrm{pp}$. William Blackwood and Sons, Edinburgh.

Nicholson, H.A. \& FoORD, A.H. 1885. On the genus Fistulipora M'Coy, with descriptions of several species. Annals and Magazine of Natural History, Series 5 16(96), 496-517. DOI 10.1080/00222938509459918

Olaussen, S. 1981. Formation of celestite in the Wenlock, Oslo
Region, Norway - evidence for evaporitic depositional environments. Journal of Sedimentary Petrology 51, 37-46.

Orbigny, A.D. D' 1850. Prodrome de paléontologie stratigraphique universelle des animaux mollusques rayonnés, faisant suite ou cours élémentaire de paléontologie et géologie stratigraphique. Volume 1. 394 pp. Victor Masson, Paris. DOI 10.5962/bhl.title.62810

Owen, D. E. 1969. Wenlockian bryozoa from Dudley, Niagara, and Gotland and their palaeogeographic implications. Palaeontology 12(4), 621-636.

Pushkin, V.I. 1975. About genus Mediapora and its new representatives from the Upper Silurian in Belarus and Latvia. Transactions of the Museum of earth-study of Moscow University 11, 113-120. [in Russian]

Pushkin, V.I. 1976. New species of Ordovician and Silurian Bryozoa of the Brest Depression, 3-40. In Akimets, V.S. \& GoveTSKII, G.I. (eds) New species of the fossil plants and animals of Belarus. Nauka i tekhnika, Minsk. [in Russian]

Pushis, V.I., Nekhorosheva, L.V., Kopaevich, G.V. \& YaroSHINSKayA, A.M. 1990. Przhydolian Bryozoa of the USSR. 125 pp. Nauka, Moscow. [in Russian]

SPJELDNÆS, N. 1982. Silurian Bryozoa of the Oslo Region. IUGS Subcommission on Silurian Stratigraphy. Field Meeting, Oslo Region 1982. Paleontological Contributions from the University of Oslo 278, 55-62.

UlRICH, E.O. 1879. Description of a new genus and some new species of bryozoans from the Cincinnatian Group. Journal of the Cincinnati Society of Natural History 2, 119-131.

UlRich, E.O. 1882. American Palaeozoic Bryozoa. The Journal of the Cincinnati Society of Natural History 5, 121-175, 233-257.

ULRICH, E.O. 1888. On Sceptropora, a new genus of Bryozoa, with some remarks on Helopora Hall, and other genera of that type. The American Geologist 1(4), 228-234.

Ulrich, E.O. 1890. Palaeozoic Bryozoa, 283-688. In Worthen, A.H. \& Lindahl, J. (eds) Geological Survey of Illinois, Vol. 8, Geology and Palaentology, Part II. Geological Survey of Illinois, Springfield.

UlRICH, E.O. 1893. On Lower Silurian Bryozoa of Minnesota. The Geological and Natural History Survey of Minnesota, final report 3, 96-332.

VINE, G.R. 1884. Fourth report of the Committee consisting of Dr. H.R. Sorby and Mr. G.R. Vine, appointed for the purpose of reporting on fossil Polyzoa, 161-209. Report of the Fifty-third meeting of the British Association for the Advancement of Science (Southport, 1883). John Murray, London.

WhitAKer, J.H.M. 1977. A Guide to the Geology around Steinsfjord, Ringerike. 56 pp. Universitetsforlaget, Oslo.

Worsley, D., Aarhus, N., Bassett, M.G., Howe, M.P.A., Mørk, A. \& Olaussen, S. 1983. The Silurian succession of the Oslo Region. Norges geologiske undersøkelse 384, 1-57.

Worsley, D., BaArli, B.G., Howe, M.P.A., Hualtason, F. \& Alm, D. 2011. New data on the Bruflat Formation and the Llandovery/Wenlock transition in the Oslo Region, Norway. Norwegian Journal of Geology 91, 101-120. 